\title{
Exercise intervention protocol in children and young adults with cerebral palsy: the effects of strength, flexibility and gait training on physical performance, neuromuscular mechanisms and cardiometabolic risk factors (EXECP)
}

Pedro Valadão ${ }^{1 *}$ D, Harri Piitulainen ${ }^{1,2}$, Eero A. Haapala ${ }^{1,3}$, Tiina Parviainen ${ }^{4}$, Janne Avela ${ }^{1}$ and Taija Finni ${ }^{1}$

\begin{abstract}
Background: Individuals with cerebral palsy (CP) have problems in everyday tasks such as walking and climbing stairs due to a combination of neuromuscular impairments such as spasticity, muscle weakness, reduced joint flexibility and poor coordination. Development of evidence-based interventions are in pivotal role in the development of better targeted rehabilitation of $\mathrm{CP}$, and thus in maintaining their motor function and wellbeing. Our aim is to investigate the efficacy of an individually tailored, multifaceted exercise intervention (EXECP) in children and young adults with CP. EXECP is composed of strength, flexibility and gait training. Furthermore, this study aims to verify the short-term retention of the adaptations three months after the end of the EXECP intervention.

Methods: Twenty-four children and young adults with spastic CP will be recruited to participate in a 9-month research project with a 3-month training intervention, consisting of two to three 90-min sessions per week. In each session, strength training for the lower limbs and trunk muscles, flexibility training for the lower limbs and inclined treadmill gait training will be performed. We will evaluate muscle strength, joint flexibility, neuromuscular and cardiometabolic parameters. A nonconcurrent multiple baseline design with two pre-tests and two post-tests all interspaced by three months is used. In addition to the CP participants, 24 typically developing age and sexmatched participants will perform the two pre-tests (i.e. no intervention) to provide normative data.

* Correspondence: pedro.valadao@jyu.fi

${ }^{1}$ Neuromuscular Research Center, Faculty of Sport and Health Sciences, University of Jyväskylä, Jyväskylä, Finland

Full list of author information is available at the end of the article

C C The Author(s). 2021 Open Access This article is licensed under a Creative Commons Attribution 4.0 International License, which permits use, sharing, adaptation, distribution and reproduction in any medium or format, as long as you give appropriate credit to the original author(s) and the source, provide a link to the Creative Commons licence, and indicate if changes were made. The images or other third party material in this article are included in the article's Creative Commons licence, unless indicated otherwise in a credit line to the material. If material is not included in the article's Creative Commons licence and your intended use is not permitted by statutory regulation or exceeds the permitted use, you will need to obtain permission directly from the copyright holder. To view a copy of this licence, visit http://creativecommons.org/licenses/by/4.0/. The Creative Commons Public Domain Dedication waiver (http://creativecommons.org/publicdomain/zero/1.0/) applies to the data made available in this article, unless otherwise stated in a credit line to the data. 
(Continued from previous page)

Discussion: This study has a comprehensive approach examining longitudinal effects of wide variety of variables ranging from physical activity and gross motor function to sensorimotor functions of the brain and neuromuscular and cardiometabolic parameters, providing novel information about the adaptation mechanisms in cerebral palsy. To the best of our knowledge, this is the first intervention study providing supervised combined strength, flexibility and gait training for young individuals with CP.

Trial registration number: ISRCTN69044459, prospectively registered (21/04/2017).

Keywords: Cerebral palsy, Strength, Flexibility, Gait, Training, Neuromuscular, Cardiometabolic

\section{Background}

\section{Cerebral palsy}

Cerebral palsy (CP) describes a group of permanent disorders of the development of movement and posture, that are attributed to nonprogressive disturbances that occurred in the developing fetal or infant brain [1]. The spastic-type CP accounts for approximately $80 \%$ of all CP cases [2-4] and is characterized by hyper-resistance [5] which has three components: stretch hyperreflexia (i.e. velocity dependent involuntary activation), involuntary background activation and altered muscle-tendon mechanical properties. Muscle-tendon unit mechanical alterations [6-8] can cause severe reduction in joint range of motion (i.e. contractures) and lead to bone deformation [9-11]. The development of contractures does not seem to be caused solely by hyperreflexia [12, 13]; rather muscle inactivity and impaired muscle growth seems to be important factors [14, 15]. Furthermore, another debilitating symptom in $\mathrm{CP}$ is a decreased muscle strength $[6,16-18]$ that severely hinders the ability to perform tasks such as walking and climbing stairs [19$21]$. These secondary clinical symptoms induce lower physical activity (PA) levels in people with CP $[22,23]$ and lead to higher levels of body adiposity and other cardiometabolic risk factors $[16,24,25]$. Therefore, although the initial brain lesion is nonprogressive in $\mathrm{CP}$, secondary symptoms such as reduced muscle strength and joint range of motion (ROM) typically further deteriorate with time, increasing sedentarism and creating a cycle of inactivity and loss of function [26, 27]. Thus, well targeted therapeutic interventions to reduce these secondary problems and maintain motor function are in pivotal role to enable independent mobility in individuals with $\mathrm{CP}$ and thus support their lifelong health and wellbeing.

\section{Strength training for $\mathrm{CP}$}

Cross-sectional studies demonstrated that muscle strength had a high shared variance with gait and motor function in CP $\left(r^{2}=50-60 \%[28,29]\right.$;). Furthermore, the effectiveness of strength training complying with known training guidelines $[25,30,31]$ to increase muscle strength in individuals with $\mathrm{CP}$ has been well demonstrated [32-40]. Nevertheless, these interventions have reported no changes [33, 35-38] or improvement in motor function [32, 34, 35]. This controversy seems to arise from two main reasons. Firstly, functional tasks involve a complex interaction between muscle strength, joint flexibility (i.e. maximum joint $\mathrm{ROM}$ ) and motor coordination. Secondly, the neural and morphological adaptations are highly specific to the strength training methods $[35,41]$, which varied in these studies. A final remark is that every day functional tasks involve activation of several muscle groups spanning across joints. Thus, an adequate intervention should train all relevant muscles with naturalistic patterns of neural activation.

A major yet unproven concern is that strength training could increase hyper-resistance and thus would be an unadvisable intervention [42]. Only few strength training studies have measured components of hyper-resistance longitudinally and reported no changes after the intervention [33, 34, 37, 38]. Furthermore, the term "spasticity" is often used under varied definitions and assessment methods [43]. For example, the modified Ashworth Scale [44] utilized in two of these studies [33, 34] is a subjective assessment of resistance to passive movement [45]. Scholtes et al. [37, 38] evaluated hyperreflexia by assessing the presence of a catch (i.e. sudden increase in muscle tone) in response to a single fast $(<1$ s) passive stretch, being also a subjective measure of the velocity-dependent resistance to passive movement. Both measurements fail to differentiate between the different components of hyper-resistance. Therefore, although it is generally accepted that strength training does not increase "spasticity" [46], no longitudinal study has reported an objective, valid and reliable measurement of hyper-resistance.

\section{Flexibility training for $\mathrm{CP}$}

Stretching is often utilized to prevent or treat loss of joint flexibility, however a recent systematic review pooling different stretch modalities (e.g. splint, serial casting, manual therapy) for different patient groups (e.g. CP, stroke, spinal cord injury) reported high-quality evidence that it does not have clinically relevant effects [47]. Passive stretching has the advantage of not restricting joint 
movement for a prolonged time as opposed to serial casting or splints, however, its effectiveness to increase joint flexibility in people with CP is unclear [48, 49]. Theis et al. [50] demonstrated that passive dorsiflexion stretching caused an acute increase in flexibility due to the elongation of both muscle and tendon. However, comprehensive longitudinal data is scarce. Theis et al. [51] showed an increase in dorsiflexion flexibility after a 6-week passive stretching intervention (4 days per week, 15 min per day). Zhao et al. [52] demonstrated that passive dorsiflexion stretching and active ankle joint movement within a 6-week intervention was able to increase fascicle length of both soleus (Sol) and medial gastrocnemius (MG). However, the study did not have a control group and the sample was composed of children, thus maturation may have been an intervening variable. The disparity between the limited scientific understanding of stretching effectiveness for $\mathrm{CP}$ and its widespread utilization must be addressed swiftly.

\section{Gait training for CP}

The inability to dorsiflex the ankle joint during the stance phase (i.e. toe walking) and swing phase of gait (i.e. foot drop) are common problems in CP [53]. A recent literature review has concluded that gait training is safe, feasible and effective to improve walking ability in children and young adults with CP [54]. The incline treadmill setup seems particularly effective as it forces a greater ankle dorsiflexion in the swing phase and serves as a dynamic passive stretch for the ankle plantarflexors in the stance phase $[55,56]$. Daily gait training with an inclined treadmill has been demonstrated to increase walking speed, dorsiflexion strength and active ROM, and reduce ankle stiffness in only four to six weeks [57, 58]. No changes in ankle passive flexibility were found in these studies, however the decrease in passive stiffness suggests that the muscle-tendon unit is adaptable, but further improvements in $\mathrm{ROM}$ may require a longer period of training. It is noteworthy that altering gait pattern and automatizing it for use in everyday over ground walking may require intense individualized feedback, so the person is aware or is at least guided towards the correct pattern (e.g. verbal feedback, biofeedback/augmented reality; [54]).

\section{Study purpose}

The present study has three aims: 1) to investigate the effects of the EXECP intervention on the following variables: gait performance (primary outcome), physical activity level, lower limb muscle strength and joint flexibility, gross motor function, cardiometabolic risk factors, neuromuscular parameters for triceps surae muscle, cortical processing of proprioceptive stimuli evoked by passive ankle dorsiflexions, tibialis anterior (TA) cortico-muscular and intramuscular coherence; 2) to evaluate the retention of the adaptations induced by the intervention after three months; 3) to compare $\mathrm{CP}$ and typically developing (TD) individuals in all studied variables using group and matched (age/sex) individual data. The EXECP intervention is composed of a three-month individually tailored exercise intervention containing strength training targeting lower limb and trunk muscles, combined with stretching of diagnosed short muscles and gait training. The primary hypothesis is that the EXECP intervention will enhance gait performance by: a) increasing walking distance in the $6 \mathrm{~min}$ walking test; b) increasing ankle dorsiflexion during the swing and stance phase of gait; c) increasing maximal walking velocity, joint net moments and ranges of motion in the lower limb joints. The secondary hypothesis are that the EXECP intervention will: d) increase habitual PA; e) increase maximal isometric and concentric torque and rate of force development for the trained muscles; f) not affect cardiometabolic risk factors such as arterial stiffness, insulin resistance, blood lipids and body fat content; g) increase gross motor function measure (GMFM; [19]) score; h) increase lower limb joint flexibility of the trained muscle groups; i) decrease ankle plantarflexors average joint stiffness and increase joint energy (i.e. area under the torque-angle curve) during slow passive stretching; $j$ ) decrease antagonist muscle electromyography (EMG) during maximal voluntary isometric and concentric plantarflexion and dorsiflexion; $\mathrm{k}$ ) not change spinal mechanisms related to hyperreflexia in the Sol muscle (i.e. Hoffmann-reflex (H-reflex), post-activation depression (PAD) ratio, tonic stretch reflex threshold (TSRT)); l) increase amplitude of cortical responses to proprioceptive stimulation, recorded with magnetoencephalography (MEG) in the primary somatosensory cortex; $m$ ) increase cortico-muscular coherence between cortical MEG and TA EMG signals; $n$ ) increase intramuscular coherence within TA muscle during a submaximal isometric dorsiflexion and during the swing phase of the gait cycle. No changes in any of the studied variables are expected during the 3months control period, except if the participant is going through the growth spurt. After the 3-month maintenance period, the values of the studied variables are expected to be in between the control and post intervention values (project aim B). All study variables are hypothesized to show differences between the TD and CP groups (project aim C). It is expected that the comprehensive assessment battery of neuromuscular, brain, cardiometabolic and functional parameters will add valuable information about how people with $\mathrm{CP}$ adapt to exercise. 


\section{Methods}

\section{Study design}

The present study utilizes a nonconcurrent multiplebaseline design $[59,60]$ composed of two pre-tests interspaced by three months (i.e. control period), followed by the three-month EXECP-intervention, and two posttests performed immediately after and three months after the intervention (i.e. maintenance period; Fig. 1). The TD age and sex-matched control group will be tested in the two pre-tests without the intervention. The nonconcurrent multiple baseline design was selected over the randomized controlled trial (RCT) because the participants inside the inclusion criteria are expected to form a highly heterogeneous group regarding the studied variables (e.g. motor control, muscle strength, joint flexibility and gait mechanics), and randomly dividing the sample into two groups is not likely to result in two comparable groups. The chosen experimental design circumvents the expected high variability in the studied variables by allowing the subjects to be their own controls. Additionally, it allows performing the EXECP intervention all year-round, avoiding systematic seasonal influences to affect the study variables (e.g. vacations, seasons [61];). During the participation in the project, both groups will perform their normal activities (i.e. physiotherapy and/or sport activities), with the instruction of maintaining the number of sessions and activity type as stable as possible. Exceptionally during the intervention period, a reduction in the volume of the activities may be necessary to avoid overloading the participants. Any important deviations from the activities done during the control period will be reported.

Eligible participants: CP Group: Children and young adults ( $9-24$ years) with spastic hemiplegia or diplegia-type cerebral palsy, GMFCS LIII. Exclusion criteria: lower limb surgery, pharmacological treatment and strength training in the past 6 months, inability to understand basic instructions, dystonia, reduced ankle ROM (unable to reach $0^{\circ}$ ), current utilization of serial casting. TD group: Typically developing children and young adults (age and sex matched).

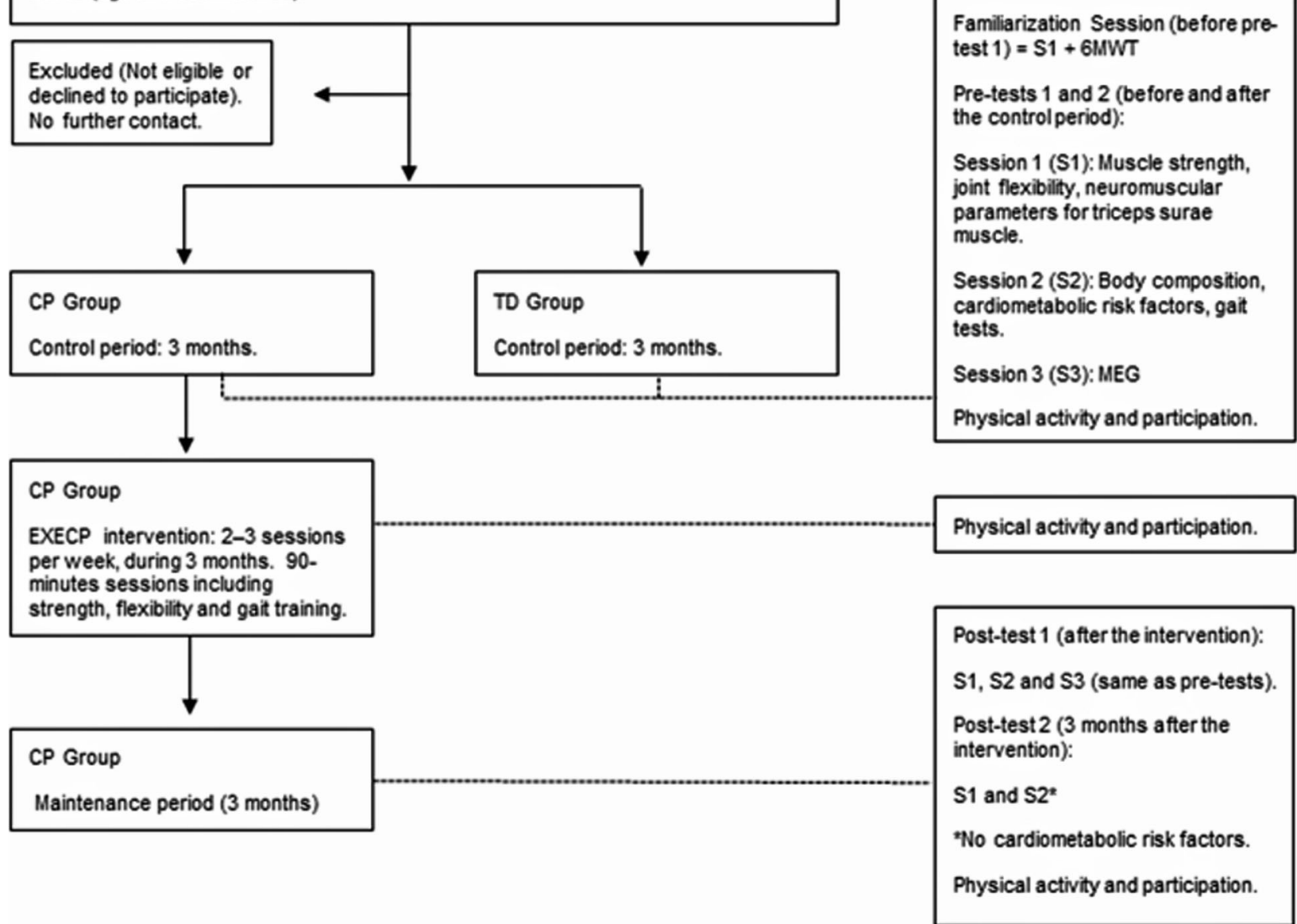

Fig. 1 Flow chart for EXECP study. CP = cerebral palsy; TD = typically developing; GMFCS = gross motor function classification system; ROM = range of motion; $6 \mathrm{MWT}$ = six minutes walking test; $\mathrm{MEG}$ = magnetoencephalography 
The first author will be responsible for all tests, and it is not possible to blind him to group allocation. Testing procedures were defined in detail to ensure consistency. The outcome data is quantitative and objective, with little possibility to suffer from observer bias. However, because the tester will obviously hope for better results in the Post-tests, no data analysis will be performed before the data collection is done (except for the flexibility tests). Thus, the tester will not have any knowledge of the results and will be focused on implementing the tests according to the protocol.

\section{Study sample and recruitment}

Twenty-four individuals aged 9-24 years with spastictype CP will be recruited predominantly in the city of Jyväskylä, Finland. Hospitals, physiotherapy clinics and $\mathrm{CP}$ associations will be contacted in search of suitable participants. Acquiring the whole sample size in Jyväskylä was not possible, so the recruitment of participants was extended to nearby cities. Furthermore, the coronavirus caused a three-month lockdown in spring 2020, which paused the participation of some participants and delayed recruitment. Twenty-four typically developing age and sex-matched controls will also be recruited from local schools and the University of Jyväskylä. The recruitment has been ongoing since May of 2017 and it is expected to end in December of 2020.

\section{Inclusion criteria}

The project will accept male and female participants who are aged 9-24 years, with a confirmed diagnosis of unilateral or bilateral spastic $\mathrm{CP}$ and are classified as level I to III in the Gross Motor Function Classification System (GMFCS [62];).

\section{Exclusion criteria}

The project will not accept participants who: a) have had lower limb surgery and/or pharmacological treatments (e.g. intrathecal baclofen, botulinum toxin) in the past six months; b) have had selective dorsal rhizotomy; c) are utilizing serial casting on the lower limbs; d) have participated in a resistance training program for the lower limbs in the last six months; e) are unable to provide sufficient cooperation in the intervention and testing sessions; f) are unable to stand with both heels touching the floor (i.e. ankle in anatomical position). Exceptionally, participants within exclusion criteria a) and b) may be accepted as case studies.

\section{Sample size}

Power calculation was performed for the project's primary hypothesis (i.e. gait performance), specifically for the effect of the EXECP intervention on walking performance measured by the six minutes walking test
(6MWT [63];). The distance covered in the 6MWT is reported to reflect functional capacity and has been recommended as a submaximal exercise test for children with CP of GMFCS I to III [64, 65]. Results from Pretests 1 and 2 for the first 9 participants in the project were utilized for sample size calculation utilizing an online spreadsheet (www.sportsci.org [66];). Data was logtransformed due to the inexistence of zero and negative values, yielding a typical error of 1.06, between-subject SD of 1.46 and a smallest important change of 1.08. Utilizing the pre-post parallel-groups RCT model, with a power of 0.8 and an alpha of 0.05 , a sample size of 24 participants in both $\mathrm{CP}$ and TD group is required. No specific sample size calculation algorithm for our experimental design was found, thus the RCT calculation served as an upper bound for the sample size.

\section{Study procedures}

The EXECP intervention will mainly take place at the NMRC, although it can be performed in other gyms having all required training equipment. It consists of two to three training sessions per week for 12 weeks. The number of weekly sessions will depend on the level of PA of each participant: those engaged in regular weekly PA may choose to perform 2 or 3 sessions, while sedentary participants will train 3 times per week. Training sessions are $90 \mathrm{~min}$ in duration and are separated by at least $48 \mathrm{~h}$. Each training session starts with an inclined treadmill walking $(5-10 \mathrm{~min})$, followed by strength $(60-$ $75 \mathrm{~min}$ ) and flexibility training $(10-20 \mathrm{~min})$. To ensure optimum training quality all sessions are performed individually and supervised by a strength and conditioning coach or a physiotherapist with full understanding of the training protocol.

\section{Gait training}

A portable mechanical treadmill with an inclination of $6^{\circ}$ or $7.3^{\circ}$ (Vida XL, Venlo, Netherlands) will be utilized for training. Participants are instructed to walk at a comfortable speed, avoiding toe walking and trying their best to attain heel strike. A non-motorized treadmill was chosen because belt movement is attained by pushing it with hip extension and ankle plantarflexion, reinforcing a correct pattern of muscle activation. Verbal feedback will be constantly given during the warm-up to improve gait quality, and the participant will be allowed to stop and rest whenever necessary. In addition to 5-10 min of gait training in every session, all participants will receive a treadmill to take home, and are asked to walk a minimum of $10 \mathrm{~min}$ per day every day for the duration of the intervention. An exercise diary will be filled by the participants or their guardians to document the weekly walking durations. During the maintenance period, the participants will choose if they want to keep using the treadmill at home and updating their diary or stop and return it. 


\section{Strength training}

Five single joint and five multi-joint exercises were selected for the intervention (Table 1). This composition was chosen because single joint exercises allows greater loading control minimizing compensatory movements $[24,64]$, whereas multi-joint exercises offer a mixture of strength and motor control training that may be beneficial for motor function. The strength training program has two protocols $(\mathrm{A} / \mathrm{B})$, with a minimum of 7 exercises targeting lower limb and trunk muscles. However, depending on the speed that exercises can be performed, which varies considerably between GMFCS levels, 7 to 10 exercises may be performed in every session. The protocols are trained weekly in the order $\mathrm{AB}$ ( 2 sessions per week) or an alternating pattern of ABA/BAB (3 sessions per week).

Ankle plantarflexors will be trained with seated and standing calf raises. Ankle dorsiflexors will be trained utilizing a rubber band resistance against the dorsiflexion movement. Additionally, TA will also be trained isometrically during a hip flexion exercise, in which the participant laying supine must flex the hip against the rubber band resistance placed on the forefoot. Manual resistance may be used instead of the rubber band in case the trainer perceives it as easier to apply the resistance during the entire ROM. The thigh muscles will be trained utilizing seated knee extensor and knee flexor machines. Additionally, lower limb muscles will be trained using the leg press and squat holding with both hands an adjustable support. A dense foam ball may be placed between the participant's knees to prevent hip adduction during leg press and squat. Exercises will be done mostly unilaterally due to strength differences between limbs (80-100\% of total training volume), except the squat which will mostly be trained bilaterally. Bilateral training may be performed if exercise technique is correct and limb loading appears similar; based on the participant's perception of fatigue/effort and the trainer's visual evaluation. Trunk and hip flexors will be trained isometrically with the hollow rocks exercise, in which the participant lies supine on the floor and must lift the legs slightly above the floor (i.e. hip and trunk flexion, knee extension). Trunk extensors will be trained isometrically using an inclined $\left(30-45^{\circ}\right)$ roman chair. Table 1 describes the positioning, target muscles and kinesiology of each exercise.

To enhance autonomy, comfort and interaction within the sessions, participants can choose the order of the exercises if this complies with the general rule of avoiding fatigue build up, which could hinder both execution quality and intensity. More specifically, this rule states that exercises for the same muscle group always must be separated by at least one different exercise (e.g. seated and standing calf raises cannot be executed consecutively). The trainer will guide this process and suggest possible sequences. Table 2 provides an example for both protocols.

The training load was devised to increase muscle strength and mass, complying with the American College of Sports Medicine and National Strength and Conditioning Association guidelines [30, 31] and specific guidelines for CP [25]. The intervention is divided in 3 blocks of 4 weeks: the first block consists of 3 sets of 8 repetitions maximum (i.e. the ninth repetition cannot be attained due to fatigue), movement duration of $6 \mathrm{~s}(3 \mathrm{~s}$ concentric and $3 \mathrm{~s}$ eccentric), 60s of rest and no muscle relaxation between repetitions. This training load has been shown adequate to increase muscle strength and mass, and it is very safe as the intensity is approximately $50 \%$ of 1 repetition maximum [67]. Furthermore, people with $\mathrm{CP}$ usually perform strength exercises with fast concentric bursts and have difficulty in controlling slow eccentric muscle actions. Thus, this protocol also tackles an important motor control deficit in CP. In the second block, the volume is maintained while the intensity is increased by reducing the concentric movement duration to $1 \mathrm{~s}$ and increasing the rest to $90 \mathrm{~s}$. In the third block, training volume and rest are maintained, but the sets are increased to four while the repetitions are decreased to 6 , concentric muscle actions are now done as fast as possible while eccentric muscle action duration is reduced to $2 \mathrm{~s}$. Thus, concentric power is trained, and the modifications permits further increases in exercise intensity. Table 2 depicts the training load progression during the intervention.

Exceptionally, the squat exercise will follow a different progression because of its inherent higher intensity (due to body weight): one to four sets of 10 repetitions with the largest attainable ROM will be performed. Movement duration is similar to the other exercises, while the rest starts at $90 \mathrm{~s}$, and will be decreased when possible to $60 \mathrm{~s}$. After the entire volume is executable with $60 \mathrm{~s}$ of rest, balance disks (Casall, Vantaa, Finland) will be placed under the participant's feet to cause instability and increase exercise difficulty, also unilateral squats may be utilized to further increase the exercise intensity.

An important aspect of isoinertial strength training is that the ability to produce torque varies throughout the joint ROM, due to the sarcomere force-length curve and the joint's lever arm [68]. Furthermore, CP joint ROM is often reduced [69] and muscle weakness is present [24]. Thus, allowing CP participants to perform the strength exercises by themselves leads to a movement with a smaller joint ROM (as compared to the total possible active $\mathrm{ROM}$ ), and the non-optimum part of the torqueangle curve is not trained. Since strength gains are generally higher at the trained angles (i.e. length-specific adaptations [70, 71];) and increasing the torque production 


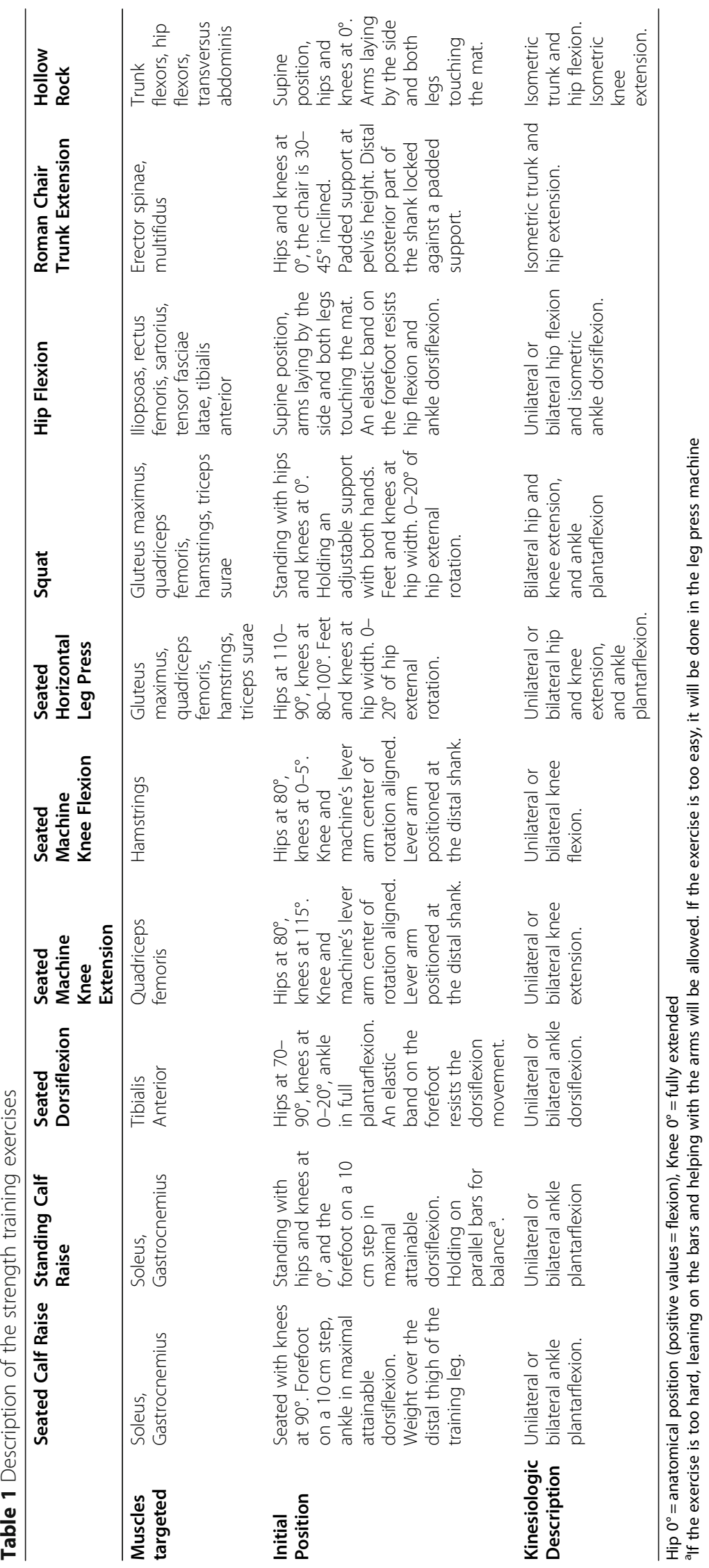


Table 2 Training progression over 12 weeks (left), and example of exercise list per training session (right)

\begin{tabular}{|c|c|c|c|c|c|c|}
\hline Week & Volume & Load & $\begin{array}{l}\text { Movement } \\
\text { Duration (s) }\end{array}$ & Rest (s) & Session $A^{a}$ & Session $B^{a}$ \\
\hline $1-4$ & 3 sets of 8 repetitions & $8 \mathrm{RM}$ & $\begin{array}{l}3 \text { concentric } \\
3 \text { eccentric }\end{array}$ & 60 & \multirow{3}{*}{$\begin{array}{l}1 \text { - Seated calf raise } \\
2 \text { - Seated dorsiflexion } \\
3 \text { - Standing calf raise } \\
4 \text { - Hip flexion } \\
5 \text { - Seated horizontal leg press } \\
6 \text { - Roman chair trunk extension } \\
7 \text { - Squat }\end{array}$} & \multirow{3}{*}{$\begin{array}{l}1 \text { - Seated machine knee flexion } \\
2 \text { - Seated machine knee extension } \\
3 \text { - Hip flexion } \\
4 \text { - Standing calf raise } \\
5 \text { - Seated horizontal leg press } \\
6 \text { - Isometric hollow rocks } \\
7 \text { - Squat }\end{array}$} \\
\hline $5-8$ & 3 sets of 8 repetitions & $8 \mathrm{RM}$ & $\begin{array}{l}1 \text { concentric } \\
3 \text { eccentric }\end{array}$ & 90 & & \\
\hline $9-12$ & 4 sets of 6 repetitions & $6 \mathrm{RM}$ & $\begin{array}{l}\text { ! concentric } \\
2 \text { eccentric }\end{array}$ & 90 & & \\
\hline
\end{tabular}

! = ballistic muscle action; $R M$ repetition maximum; ${ }^{a}=$ each session has a minimum of 7 exercises and a maximum of 10 (i.e. all exercises)

in the whole active curve seems very reasonable to allow functional benefits, an assisted training procedure will be adopted. The trainer will actively help the participant on the concentric phase of the movement in the positions in which the participant is not able to perform by himself. The exercise resistance will be selected based on the participant's strength on the optimal angles, thus the trainer will always be assisting on non-optimal angles, and never increasing the resistance. The eccentric phase will be performed unassisted, but constant feedback about movement velocity will be given. Due to the assistance during every repetition, no 8 or 6 repetition maximum test will be performed to adjust the weight, rather the trainer will frequently ask the participant to try and perform one more repetition, if successful, the weight will be increased for the next set. Whenever a participant is unable to perform a minimum concentric $\mathrm{ROM}$, the protocol will be adjusted to include an isometric muscle action of $3 \mathrm{~s}$ in each repetition. Thus, it will be a small concentric movement, followed by the isometric hold phase and then the eccentric phase. If during the intervention the concentric movement increases, the isometric part will be removed. At the start of each training session, the trainer will ask the participant if any adverse events were experienced after the previous session. Participants will be constantly reminded to provide immediate feedback about any pain or discomfort during the training sessions.

\section{Flexibility training}

Four sets of $45 \mathrm{~s}$ passive-static stretching at the pain threshold (i.e. position in which the participant acknowledges an initial stretch pain sensation) will be performed for each muscle group diagnosed short in the pre-tests. One and two-joint hip flexors will be stretched in the Modified Thomas Test position [72]. The participant lies supine on a table holding one of the lower limbs in full hip flexion (assistance may be provided), while the other leg hangs outside the table (i.e. hip extension). The only difference between the stretches is that the trainer will apply the hip extension torque at the distal thigh with the knee joint positioned either in full flexion (two-joint stretch) or in a relaxed position (one-joint stretch). The seated butterfly stretch will be utilized to stretch the hip adductors. The participant will have his back supported on a wall, hips externally rotated, knees flexed and the soles of the feet in contact. The trainer will use his knees to keep the participant's feet in position while pressing down the thighs causing hip abduction. Hamstring stretch will be performed in supine position, the trainer will secure the untrained leg flat against the floor, then flexes the participant's hip approximately to $90^{\circ}$ and applies a knee extension torque at the posterior aspect of the shank. No flexibility training will be performed for the triceps surae muscle group because of the following reasons: 1) lack of feasibility to execute the selected stretching protocol bilaterally for all tested muscles (session duration would greatly exceed $90 \mathrm{~min}$ ); 2) the inclined gait training forces the ankle joint into dorsiflexion during the stance phase, working as a passive dynamic stretch. To check if the gait intervention by itself can change flexibility, it seemed reasonable to avoid stretching triceps surae and to focus on the other muscle groups.

\section{Testing sessions}

Participants will attend the NMRC and the Centre for Interdisciplinary Brain Research (CIBR) at the University of Jyväskylä for all testing sessions. Each testing point (pre-test 1 and 2, post-test 1) consist of three testing sessions (S1, S2 and S3) performed $4 \mathrm{~h}$ to seven days apart. Exceptionally, post-test 2 is composed only of S1 and a modified S2. Session 1 (S1) consists of the following tests: joint flexibility, electrostimulation (H-reflex and PAD), muscle strength and tonic stretch reflex threshold. Session 2 (S2) consists of the following tests: blood sampling, body composition and height, arterial stiffness, instrumented gait analysis, six minutes walking test. In session 3 (S3) magnetoencephalography measurements are performed. The modified S2 performed in post-test 2 consists only of the gait tests and height measurement. Table 3 describes the timeline of each testing session. The TD group will only perform the pre-tests 1 and 2 . A familiarization session will be performed before the pretest 1 to get the participants acquainted with the tests. 
Table 3 Testing sessions timetable and procedures

\begin{tabular}{|c|c|c|}
\hline Testing session 1 (S1) & Testing session 2 (S2) & Testing session 3 (S3) \\
\hline 08.00 - Flexibility tests (Hip and Knee) $)^{\mathrm{b}}$; & 08.00 - Blood sampling; & 08.00 - Participant preparation; \\
\hline 08.15 - H-reflex/PAD protocol for Sol ${ }^{a}$ at rest; & 08.10 - Body composition, height and arterial stiffness; & 08.45 - Resting-state; \\
\hline 08.40 - Flexibility test (ankle) ${ }^{\mathrm{b}}$; & 08.30 - Breakfast; & 09.05 - Proprioceptive stimulation for $\mathrm{TS}^{\mathrm{a}}$; \\
\hline 08.50 - Preparatory activity $\mathrm{PF}^{\mathrm{a}} / \mathrm{DF}^{\mathrm{a}}$; & 09.00 - Participant preparation; & 09.30 - Preparatory activity DF; \\
\hline $09.00-\mathrm{MVC}\left(\mathrm{PF}^{\mathrm{a}} / \mathrm{DF}^{\mathrm{a}}\right)$ & 09.50 - Gait preparatory activity; & 09.40 - Isometric DF MVC; \\
\hline 09.20 - TSRT protocol $\left(\mathrm{TS}^{\mathrm{a}}\right)$ & $09.55-6 \times 1$ min gait test; & 09.50 - Cortico-muscular coherence protocol ${ }^{a}$ \\
\hline 09.30 - Preparatory activity $\mathrm{KE}^{\mathrm{a}} / \mathrm{KF}^{\mathrm{a}}$; & 10.10 - Rest; & 10.01 - Session ends. \\
\hline $09.40-M V C\left(K E^{a} / K F^{a}\right)$ & $10.25-6$ min walking test; & \\
\hline 10.00 - Session ends. & 10.31 - Session ends. & \\
\hline
\end{tabular}

NMRC Neuromuscular Research Center, CIBR Centre for Interdisciplinary Brain Research, PAD post-activation depression, Sol soleus muscle, MVC maximum voluntary contraction, PF plantarflexion, DF dorsiflexion, TSRT tonic stretch reflex threshold, TS triceps surae muscle, KE knee extension, $K F$ knee flexion; ${ }^{a}$ most affected limb is tested; ${ }^{b}$ both limbs are tested

Additionally, this session will be utilized to duplicate some tests of S1 and S2 for a repeatability study.

\section{Primary outcome measure}

The six minutes walking test will be performed on an indoor $30 \mathrm{~m}$ rubber track, and its primary result is the distance that the participant is able to walk in $6 \mathrm{~min}$. Heart rate (Polar Electro Oy, Kempele, Finland) will be measured to quantify exercise intensity (i.e. mean, maximum and minimum). An inertial measurement unit (NGIMU, $\mathrm{X}-\mathrm{IO}$ Technologies, UK) will be firmly strapped at the participant's lower back slightly above the posterior superior iliac spine. Gait variability will be calculated with refined composite multiscale entropy [73, 74] and refined multiscale permutation entropy $[75,76]$. Threedimensional (3D) raw acceleration signals and the resultant acceleration will be utilized for this calculation. Following the approach by Ihlen et al. [77], entropy will be calculated with coarseness scales of $\tau=1$ to 20 , the length of the template for entropy evaluation equals four and the tolerance for refined composite multiscale entropy will be set at 0.3 times the standard deviation of the entire test. Raw signals will be sampled at $100 \mathrm{~Hz}$, and stored on a SD card for later offline analysis utilizing a freely available java implementation (https://github. com/tjrantal/javaMSE). Cross-sample entropy [78, 79] will be utilized to compare the pre and post-tests for each participant.

\section{Secondary outcome measures}

\section{Gait analysis (kinematics, kinetics, gait variability)}

3D lower limb kinematics will be captured utilizing a Vicon system (Vicon Motion Systems, Oxford, UK), consisting of 8 cameras (MXT40) with a sampling frequency of $200 \mathrm{~Hz}$. Reflective markers will be placed at: (1) medial and lateral malleolus; (2) second metatarsal head; (3) heel; (4) lateral aspect of the shank; (5) medial and lateral femoral epicondyles; (6) three non-collinear markers at the lateral aspect of the thigh; (7) anterior superior iliac spine; (8) posterior superior iliac spine. This marker set was chosen to allow calculation of both direct kinematics with the Vicon Nexus 2.5 lower limb gait plug-in [80, 81] and inverse kinematics with the free opensource OpenSim software (http://opensim.stanford.edu/; [82]). Ground reaction forces will be simultaneously acquired at $1 \mathrm{kHz}$ using two force plates $(51 \mathrm{~cm} \times 46 \mathrm{~cm}$, AMTI OR6-6-2000, Watertown, USA) on a $7.4 \mathrm{~m} \times 0.6$ $\mathrm{m}$ rubber walking path. Inverse dynamics will be utilized to calculate joint moments using both OpenSim and Vicon Nexus for comparison purposes. Gait variability will be calculated utilizing the same procedures previously explained in the 6MWT. Gait cycles will be identified using footswitches (Noraxon, Scottsdale, USA) with two sensors placed bilaterally on the heel and forefoot.

The test consists of 6 trials of $1 \mathrm{~min}$, in which participants must walk back and forth at a maximum safe and controlled walking speed. Participants are instructed to disregard the force plates (i.e. not modify the gait to step on them) and to inform in case they are feeling tired. A preparatory activity of $2 \mathrm{~min}$ with progressively higher walking speeds will be performed before the test. Rest periods of 1-2 min between trials based on the participant's feedback will be given. Each trial can be stopped at any time for adjustments or resting, nonetheless a total of 6 min of walking will be recorded.

\section{Muscle strength}

Maximal isometric and concentric ankle plantarflexion and dorsiflexion will be assessed using a custom-build motor driven dynamometer (NMRC, University of Jyväskylä, Finland). Participants will be seated with the knee joint fully extended, hip joint flexed at $60^{\circ}$ (anatomical position $=0^{\circ}$ ), and the ankle joint at an initial position of $0^{\circ}$ (i.e. the sole of the foot at right angles to the tibial axis) or $28^{\circ}$ into plantarflexion. The foot will be firmly attached to a footplate mounted on the rotation 
platform so that the rotation axes of the ankle joint and the motor-driven platform coincide. Participants will be securely stabilized by an assembly of straps that fastened both shoulders and connected to a waist belt. An additional strap with a foam support prevents the knee joint of the tested leg from flexing. The torque around the rotational axis of the motor will be measured by a piezoelectric crystal transducer (Kistler Holding, Winterthur, Switzerland), and the ankle joint angle will be monitored by a linear potentiometer. Furthermore, a small stiff metal wire attached to a spring system, located under the calcaneus, will continuously monitor heel displacement from the footplate. Torque, joint angle, and heel displacement signals will be sampled at $1 \mathrm{kHz}$ with a 16bit A/D converter (CED power 1401, Cambridge Electronics Design, Cambridge, UK). The plantarflexion test starts with a $2 \mathrm{~s}$ maximal isometric muscle action at $0^{\circ}$, followed by an isokinetic $\left(14^{\circ} / \mathrm{s}\right)$ concentric effort until $28^{\circ}$. The dorsiflexion test starts with a $2 \mathrm{~s}$ maximal isometric muscle action at $28^{\circ}$, followed by an isokinetic $\left(14^{\circ} / \mathrm{s}\right)$ concentric effort until $0^{\circ}$. The highest attained torque at $0^{\circ}, 5.5^{\circ}, 11^{\circ}, 16.5^{\circ}, 22^{\circ}$, and $27.5^{\circ}$ among the trials will be used for analysis. This approach will be used because force fluctuations during the trials (i.e. short moments of relaxation) often happen when testing the $\mathrm{CP}$ group, thus it may be that one single trial will not have all the peak torque values for all joint angles.

Maximal isometric and concentric knee flexion and extension will be assessed using a custom-build motor driven dynamometer (NMRC, University of Jyväskylä, Finland). Participants will be seated with the knee joint fully extended $\left(0^{\circ}\right)$ or at $75^{\circ}$ of flexion and hip joint flexed at $80^{\circ}$. The distal part of the shank will be secured with a velcro strap to a strain gauge (NMRC, University of Jyväskylä, Finland) capable of measuring both tensile and compressive forces. The position of the strain gauge on the lever arm is adjustable and the distance from the axis of rotation will be individually recorded and reproduced for all following measurements. The rotation axis of both dynamometer and knee will be carefully aligned during a submaximal voluntary muscle action, to take into account the system's compliance. Participants will be securely stabilized by an assembly of straps fastening both shoulders and connected to a waist belt. The knee flexion test starts with a $2 \mathrm{~s}$ maximal isometric muscle action at $0^{\circ}$, followed by an isokinetic $\left(15^{\circ} / \mathrm{s}\right)$ concentric effort until $75^{\circ}$. An examiner will keep strong downward pressure at the distal part of thigh to prevent hip flexion during the trial. The knee extension test starts with a $2 \mathrm{~s}$ maximal isometric muscle action at $75^{\circ}$, followed by an isokinetic $\left(15^{\circ} / \mathrm{s}\right)$ concentric effort until $0^{\circ}$. Torque will be obtained by multiplying the moment arm by the force, and joint angle will be monitored by a linear potentiometer. Both signals will be sampled at $1 \mathrm{KHz}$ with the same hardware and software utilized for the ankle torque tests. The highest attained torque in nonoverlapping $5^{\circ}$ steps among all trials will be used for analysis. For all four strength tests, rate of force development will be calculated as the maximum slope during the 0 $200 \mathrm{~ms}$ time period in the isometric part. All measurements will be performed on the most affected leg for the $\mathrm{CP}$ group, and on the corresponding leg on the matching control participant. Test isokinetic velocity was chosen based on the slowest intervention training velocity $(3 \mathrm{~s}$ muscle action for the ankle joint with a ROM of $\sim 45^{\circ}$ ), and because slower velocities are easier to be well performed by the CP participants (observed in the pilot experiment). A preparatory activity consisting of ten progressively stronger efforts from 20 to $90 \%$ of the maximum voluntary contraction (MVC) will be performed before each test. Visual torque feedback will be provided in real-time and participants will receive strong verbal encouragement during every trial. Three to five trials with 1-2 min of rest for each test will be performed. In addition to the specific torque-angle analysis described, joint angles in which torque is greater than $90 \%$ of the peak concentric torque (i.e. optimum angle; [83]) and greater than $50 \%$ of the peak concentric torque (i.e. curve width; [84]) will also be calculated.

\section{Electromyography}

Three EMG setups will be utilized in the project. The first setup will be used for the strength, ankle flexibility and neural tests (i.e. H-reflex, PAD and tonic stretch reflex threshold). EMG activity will be recorded from Sol, MG and TA muscles with self-adhesive electrodes (Blue Sensor N, $\mathrm{Ag} / \mathrm{AgCl}, 0.28 \mathrm{~cm}^{2}$; Ambu, Ballerup, Denmark), and a ground electrode placed on the tibia. Electrode placement and skin preparation will be performed according to SENIAM [85]. The electrodes will be placed on the muscle belly in accordance with the underlying fiber direction (20-mm-interelectrode distance). EMG signals will be amplified and high-pass filtered $(1000 \mathrm{X}, 10 \mathrm{~Hz})$ by a preamplifier (NL824; Digitimer, Welwyn Garden City, UK) and then bandpass filtered $(10 \mathrm{~Hz}$ to $1 \mathrm{kHz})$ by a differential amplifier (NL900D/NL820A; Digitimer Ltd., UK). The signals will be acquired on a personal computer at a rate of $5 \mathrm{kHz}$ via a 16-bit A/D converter (CED power 1401; Cambridge Electronics Design, Cambridge, UK). EMG during the maximal voluntary plantarflexion and dorsiflexion trials will be quantified in $200 \mathrm{~ms}$ root mean square (RMS) windows preceding the joint angles of $0^{\circ}, 5.5^{\circ}, 11^{\circ}, 16.5^{\circ}$, $22^{\circ}$ and $27.5^{\circ}$. TA coactivation during plantarflexion will be expressed as \% of the maximal RMS EMG obtained on the dorsiflexion trial at the same joint position. The same procedure will be performed to express Sol and MG coactivation during ankle dorsiflexion. 
The second setup utilizes a wireless EMG system (Noraxon, Scottsdale, USA) that will acquire EMG activity during the instrumented gait analysis. Data acquisition configuration, muscles tested, and electrode placement are identical to setup 1, except for two differences: 1) sampling frequency is $1.5 \mathrm{kHz} ; 2$ ) two sets of bipolar electrodes will be placed in TA muscle, $8-10 \mathrm{~cm}$ apart depending on the subject's tibia length.

The third EMG setup is built-in the MEG System (Elekta Neuromag TRIUX, Elekta Oy, Helsinki, Finland). The same bipolar electrode placement as in setup 2 will be utilized, with a MEG compatible electrode (Neuroline 720, Ag/AgCl, $0.28 \mathrm{~cm} 2$; Ambu, Ballerup, Denmark). EMG signal will be sampled at $1 \mathrm{KHz}$ and band-pass filtered $1-1000 \mathrm{~Hz}$.

\section{Neural parameters}

$\mathrm{H}$-reflexes and M-waves will be evoked in Sol by percutaneous electrical stimulation of the tibial nerve, while the participant lies in prone position. A single rectangular pulse $(1 \mathrm{~ms})$ will be delivered from a constantcurrent stimulator (DS7AH; Digitimer, UK). A circular cathode with a pickup area of $0.77 \mathrm{~cm}^{2}$ (Unilect4535M, $\mathrm{Ag} / \mathrm{AgCl}$; Unomedical, Redditch, UK) will be placed over the tibial nerve on the popliteal fossa, and an ovalshaped, $5.1 \times 10.2 \mathrm{~cm}$ anode (V-trodes; Mettler Electronics, Anaheim, CA) will be placed above the patella. The stimulation site providing the greatest amplitude of evoked responses in Sol will first be located by a handheld cathode electrode that will be later replaced by a self-adhesive electrode. The full recruitment curve will be attained by starting with a stimulation intensity of 3 $\mathrm{mA}$ and increasing in $0.5-2 \mathrm{~mA}$ steps (i.e. smaller steps closer to the maximum $\mathrm{H}$-reflex) at $0.1 \mathrm{~Hz}$ until the Maximal M-wave is reached. Peak-to-peak values for maximum $\mathrm{H}$-reflex and $\mathrm{M}$-wave will be computed.

The PAD protocol will be performed after the recruitment curve with the participant in the same position. Stimulation intensity will be first adjusted to evoke $\mathrm{H}$ reflex responses corresponding to $75 \%$ of maximum $\mathrm{H}$ reflex at $0.1 \mathrm{~Hz}$, then sixteen stimuli at two frequencies $(0.1$ and $1 \mathrm{~Hz})$ will be performed. H-reflexes will be normalized by the preceding $M$-wave, and PAD will be computed as the ratio between $1 \mathrm{~Hz}$ and $0.1 \mathrm{~Hz}$ (i.e. greater values means less PAD). This normalization procedure is important because the $\mathrm{H}$-reflex is directly influenced by the preceding $\mathrm{M}$-wave (i.e. effective stimulation intensity). However, since the stimulation intensity is constant and supposedly so is the preceding M-wave, previous studies [86, 87] have chosen to report the ratio between the absolute peak-to-peak H-reflex values. Thus, for comparison purposes the absolute PAD ratio will also be calculated.
Stretch hyperreflexia of Sol and MG will be assessed utilizing the TSRT $[88,89]$. The participant will be seated with the knee joint fully extended and the ankle dynamometer previously described will induce stretches (from $20^{\circ}$ of plantarflexion to $0^{\circ}$ ) on triceps surae at four different velocities: 50, 100, 200 and $300^{\circ} / \mathrm{s}$. The stretch velocities will be delivered in a pseudo-randomized and balanced order, yielding 10 stretches in each velocity, with an interval of 2.6-2.9 s. EMG data will be used to determine the joint angle in which the stretch reflex onset occurred (i.e. dynamic stretch reflex threshold, DSRT). A regression line based on a first-order linear equation using all DSRT will be calculated on a stretch velocity-joint angle plot. From the equation, the coefficient of determination, slope and intercept with the $\mathrm{x}$ axis will be attained. The TSRT is the angle at which the regression line intersects with the $\mathrm{x}$-axis, larger values indicate a high level of stretch hyperreflexia. TSRT will be calculated separately for each muscle. In addition to the already established TSRT calculation, we propose using the latency of the H-reflex to take into account the time between the stretch reflex onset and the EMG burst onset. A typical H-reflex latency of $35 \mathrm{~ms}$ and a stretch velocity of $300^{\circ} / \mathrm{s}$ can cause a difference of $10.5^{\circ}$ between the stretch reflex and EMG onset, provoking a systematic error towards higher velocities. Thus, all variables in the TSRT will be calculated with and without the latency correction, and the differences will be discussed. Participants will be instructed to relax and will be wearing noise blocking headphones. Trials with EMG exceeding $5 \%$ of the isometric plantarflexion MVC in the $500 \mathrm{~ms}$ preceding the stretch will be discarded. The EMG burst onset will be defined as a 3SD increase in the RMS of a $20 \mathrm{~ms}$ moving average for at least $20 \mathrm{~ms}$ compared to the pre-stretch state. Visual inspection of false positives due to small brief muscle activations will be performed and automated onset detection algorithms may also be utilized. H-reflex, PAD and TSRT will be assessed on the most affected limb of the $\mathrm{CP}$ group and in the same leg of the age/sex matched control.

Frequency domain analysis of the EMG will be performed in the following conditions: A) intramuscular TA coherence during the swing phase of gait; B) corticomuscular and intramuscular TA coherence during a sustained isometric ankle dorsiflexion. Condition A will be collected with EMG setup 2, during the instrumented gait analysis test $(\mathrm{S} 2)$, and $\mathrm{B}$ will be collected in the MEG session (S3), using EMG setup 3. Surface EMG will be preprocessed using full-wave rectification as it has been shown to be appropriate for low force muscle actions [90-92]. The finite Fourier Transform using the method of disjoint sections will be utilized [93], in which the complete EMG record R (i.e. approximately $60 \mathrm{~s}$ for $\mathrm{A}$, and $480 \mathrm{~s}$ for $\mathrm{B}$ ) is divided in $\mathrm{L}$ non-overlapping 
disjoint sections of length $\mathrm{T}$, where $\mathrm{R}=\mathrm{LT}$. For $\mathrm{A}$, $\mathrm{T}$ will be selected to reach a spectral resolution $\leq 5 \mathrm{~Hz}$. If possible, the swing phase will be divided into two or three parts, depending on the actual length of these phases and the achieved frequency resolution. For $B, R=480 \mathrm{~s}$ and $\mathrm{T}=1 \mathrm{~s}$, yielding a frequency resolution of $1 \mathrm{~Hz}$. The coherence between two rectified EMG signals ( $x$ and $y$ ) is defined as their cross-spectrum normalized by the auto-spectra for a given frequency $(\lambda)$, according to the following equation:

$$
\left|R_{x y}(\lambda)\right|^{2}=\frac{\left|f_{x y}(\lambda)\right|^{2}}{f_{x x}(\lambda) f_{y y}(\lambda)}
$$

Coherence measures how closely two signals are related by a linear transformation [93, 94], providing a bounded and normative measure of association, taking values between 0 and 1 . A perfect linear relationship has a value of 1 , while full independence has a value of 0 . Pooled coherence estimates will be calculated to characterize both control and experimental groups [95]. The estimation of pooled coherence joins separate signals from different subjects into a single longer signal, and then calculates the coherence of this record using the periodogram approach. Pooling the EMG signal of different participants may result in a non-constant standard deviation (i.e. nonstationarity) between the different sections, in this case signals will be normalized to have unit variance [96].

\section{Joint flexibility and triceps surae mechanics}

Lower limb joint flexibility will be assessed using three tests: the modified Thomas test, the passive knee extension test and a passive ankle dorsiflexion test. In the modified Thomas test, three measurements are performed at the hip joint and 2 at the knee joint [72]. The first hip measure has the goniometer fulcrum at the greater trochanter of femur, reference lines using lateral femoral condyle and a line perpendicular to the bench. The second hip measurement is like the first, however the researcher will apply a hip extension torque at the distal thigh and will record the angle at which the subject reports initial stretch pain sensation. The third hip measure has the goniometer fulcrum at the anterior superior iliac spine, references lines using the superior aspect of the patella and a line parallel to the bench. The first knee measure has the goniometer fulcrum at the lateral femoral condyle, reference lines using lateral malleolus and greater trochanter of femur. A limitation of the knee angle to access two-joint hip flexors flexibility is that it is directly affected by the hip angle 1 (one-joint hip flexors). Thus, the second knee angle is measured with the participant in the same position, except that the thigh of the tested leg is supported on the bench (instead of hanging outside of it), thus hip angle will be $0^{\circ}$. It should be noted that only hip measurement 2 is a flexibility test, because attaining maximum ROM requires unlimited torque and the subject acknowledging stretch sensation pain. On the other four measurements, the joint angle attained is a result of the interaction between gravity and the joint's passive resistance to stretch. Thus, a stiffer joint will not yield much to the gravity force, and the reduced ROM cannot be mistakenly interpreted as poor flexibility. Although not true flexibility tests, these measurements provide important information about how different muscles affects the lower limb joints.

The passive knee extension test is performed in the same position as the hamstring stretch with the participant lying supine with hip and knees at $90^{\circ}$. The nontested thigh is strapped to the bench to avoid pelvic retroversion during the test. The examiner slowly applies torque at the posterior aspect of the shank causing knee extension, while another examiner maintains the hip position. The test stops when the participant acknowledges an initial stretch pain sensation. A similar knee angle measurement used in the modified Thomas test is performed. A manual goniometer is utilized to perform the both tests.

The passive dorsiflexion test will be performed using the same motor-driven dynamometer utilized for the ankle strength tests. The participant will seat with the hip joint flexed at $60^{\circ}$, ankle at $35.5^{\circ}$ of plantarflexion and knee on two possible positions: $90^{\circ}$ or $0^{\circ}$. The examiner will slowly $\left(<5^{\circ} / \mathrm{s}\right)$ move the device causing ankle dorsiflexion until the participant acknowledges an initial stretch pain sensation. Another criterion to stop the test is a heel raise higher than $5 \mathrm{~mm}$. Since the motor-driven dynamometer will be also utilized subsequently to perform very fast dorsiflexions for the TSRT test, for safety reasons the maximum attained dorsiflexion angle is $21^{\circ}$. Thus, it is possible that maximal ROM is not measurable for some participants in the extended knee position. Three to five trials in each knee position will be performed and the maximum ROM for each position and leg will be reported. From the passive dorsiflexion torque-angle curve, in the region encompassing 20$80 \%$ of the participant's peak torque [51], average joint stiffness (i.e. first derivative), energy (i.e. integral) and hysteresis (difference between loading and unloading integral) will be calculated. Additionally, these variables will be calculated utilizing absolute joint angles. Trials with triceps surae EMG RMS higher than $3 \mathrm{SD}$ of resting levels will be discarded (EMG setup 1). Table 4 presents the criteria for each joint flexibility measurement based on TD normative data [97-103]. 
Table 4 Joint flexibility tests and references from typically developing controls attained in other studies

\begin{tabular}{l} 
Reference Values and Diagnostic \\
\hline Modified Thomas test \\
Hip measurement 1: Hip angle $>0^{\circ}=$ short and/or stiff one-joint hip \\
flexors (i.e. iliopsoas, hip adductors). \\
Hip measurement 2 (examiner applies hip extension torque): Hip \\
angle $>0^{\circ}=$ short one-joint hip flexors. \\
Hip measurement 3: Reference value $=0^{\circ}$ (i.e. ASIS and patella aligned \\
on the sagittal plane). Positive values $=$ short and/or stiff hip \\
abductors. \\
Knee measurement 1: Knee angle $<67^{\circ}=$ short and/or stiff two-joint \\
hip flexors (i.e. rectus femoris, tensor fascia latae, sartorius). \\
Knee measurement 2 (supported hip): Knee angle $<67^{\circ}=$ short and/ \\
or stiff two-joint hip flexors. \\
Note: Tightness of the anterior capsule and ligaments may also \\
influence the test. \\
References: $[72,97-99]$ \\
Passive knee extension test \\
Knee angle $>40^{\circ}$ (with the hip at $90^{\circ}$ ) $=$ short hamstring muscles. \\
References: $[100,101]$ \\
Passive ankle dorsiflexion test \\
Ankle angle $<18^{\circ}$ with knee at $0^{\circ}$ and $90^{\circ}=$ short Soleus muscle. \\
Ankle angle $<18^{\circ}$ with knee at $0^{\circ}$ and ankle angle $>18^{\circ}$ with knee at \\
$90^{\circ}=$ short Gastrocnemius muscle. \\
Ankle angle $>18^{\circ}$ in with knee in $0^{\circ}$ and $90^{\circ}=$ good triceps surae \\
flexibility. \\
References: $[102,103]$. \\
\hline Asl anteror sup
\end{tabular}

ASIS anterior superior iliac spine. Hip measurements 1-2: $0^{\circ}=$ anatomical position, positive values $=$ hip flexion. Knee measurements: $0^{\circ}=$ knee in full extension. Ankle measurements: $0^{\circ}=$ sole of the foot at right angles to the tibial axis, positive values $=$ dorsiflexion

Neuromechanical responses during the TSRT protocol (i.e. 40 stretches at 4 velocities) will also be analyzed. The higher stretch velocities in this protocol will result in reflex muscle activation, thus involving both passive and active elements. First, mechanical artifacts at the beginning and end of each stretch will be removed, then peak and mean torque, joint stiffness and energy will be calculated for each stretch. Additionally, maximum EMG RMS (EMG setup 1) will be calculated with a 50 ms moving window for each stretch. Comparisons between velocities and between the first and last group of 8 stretches will be performed to gain insight into the differences in neuromechanical responses to dynamic repetitive stretching.

\section{Physical activity}

PA will be assessed using questionnaires and devicebased measurements. The questionnaire from Jackson et al. [104] with a scale from 0 to 7 describing the overall PA level is complemented by questions about the amount of moderate-to-vigorous PA during a week. PA will also be measured with a tri-axial waist-worn accelerometer (X6-1a, Gulf Coast Data Concepts Inc., Waveland, MS, USA) with a dynamic range of $\pm 6 \mathrm{~g}$ and a sampling frequency of $40 \mathrm{~Hz}$. The accelerometer will be utilized during seven typical days at three time points: control, intervention and maintenance periods. A minimum of 3 days with $8 \mathrm{~h}$ of measurement will be accepted for analysis as it has been shown to provide adequate reliability for children and adolescents with CP GMFCS I [105]. Raw acceleration data will be transformed to Actigraph counts [106], in order to utilize validated GMFCS specific thresholds for different intensity zones for children with CP [107].

Specialized shorts with textile electrodes (Myontec Ltd., Kuopio, Finland, 108) will be utilized to record EMG bilaterally from quadriceps and hamstring muscles during an entire typical day. A small recording electronic module is able to collect muscle activity data for $8-12 \mathrm{~h}$ and can be analyzed offline afterwards. EMG signals will be acquired with a sampling frequency of $1 \mathrm{KHz}$ and band-pass filtered $(50-200 \mathrm{~Hz},-3 \mathrm{~dB})$. The raw signals will be full wave rectified and averaged over $100 \mathrm{~ms}$ nonoverlapping windows and stored into a portable module. Daily muscle inactivity, light, moderate and vigorous activity time of the participant will be extracted [108, 109]. Muscle inactivity will be defined as $<90 \%$ of the standing EMG activity for each muscle group. Moderate activity threshold is the preferred walking EMG activity, and vigorous activity is twice the amplitude of walking [110]. The participants will receive a diary to record times when the accelerometer and EMG pants are worn, facilitating the removal of non-wear time in the analysis.

\section{Gross motor function}

Gross motor function will be assessed in the CP group using the 66-item version of the Gross Motor Function Measure (GMFM-66; [111]). GMFM-66 is a valid, reliable and responsive observational instrument, based on interval scaling to evaluate changes in gross motor function in participants with CP [19]. Dimensions D (standing) and $\mathrm{E}$ (walking, running and jumping) will be utilized. The test will be video recorded and scored subsequently by the same evaluator. Absolute and relative (i.e. percentage of maximum) scores for each dimension will be reported.

\section{Biological maturity}

To control for the confounding effect of maturation on the dependent variables during the study, estimated time from peak height velocity (i.e. maturity offset) will be computed using the sex-specific equation provided by Moore et al. [112]: 


$$
\begin{aligned}
\text { Girl's maturity offset }= & -7.709133 \\
& +(0.0042232 *(\text { age } * \text { height })) ; \mathrm{R}^{2} \\
= & 0.898 ; \mathrm{SEE}=0.528
\end{aligned}
$$

Boy's maturity offset $=-7.999994$

$$
+(0.0036124 \times \text { (age } \times \text { height }), R^{2}
$$$$
=0.896 ; \mathrm{SEE}=0.542 \text {. }
$$

\section{Body composition, height and cardiometabolic risk factors}

Body height will be measured with the head positioned in the Frankfurt plane with a wall-mounted stadiometer and body composition with a bioelectrical impedance device (InBody 720, Seoul, South Korea; [113]). Venous blood samples will be drawn after standard overnight (12 h) fast. Serum insulin will be measured by electrochemiluminescence immunoassay (Immulite 2000, Siemens, Germany). Fasting plasma glucose, total plasma cholesterol, plasma high and low-density cholesterol and plasma triglycerides will be measured by enzymatic colorimetric assays (Konelab 20XTi, Thermo, USA). Insulin sensitivity estimated by the Homeostatic Model Assessment for Insulin Resistance (HOMA-IR) will be calculated using the formula: fasting serum insulin $\mathrm{x}$ fasting plasma glucose/22.5 [114]. White and red blood cells, hematocrit and hemoglobin will be measured by an automated analyzer (Sysmex XP300, Sysmex, Japan). Systolic and diastolic blood pressure, aortic pulse wave velocity, and augmentation index will be measured in supine position with a non-invasive oscillometric device after $10 \mathrm{~min}$ of rest (Arteriograph, Tensiomed Ltd., Budapest, Hungary; [115]). Participants will be allowed to drink water and take their usual medications during fastening.

\section{Magnetoencephalography (MEG)}

Participants will be seated inside a magnetically shielded room (VACOSHIELD, Vacuumschmelze $\mathrm{GmbH} \&$ Co. KG, Hanau, Germany) with their heads positioned within the sensor array of the whole-head-MEG System (Elekta Neuromag TRIUX, Elekta Oy, Helsinki, Finland), consisting of 204 planar gradiometers and 102 magnetometers. MEG data will be acquired at $1000 \mathrm{~Hz}$ and band-pass filtered $(0.1-330 \mathrm{~Hz})$.

Subject preparation Five head position indicator (HPI) coils will be attached to the participant's scalp to measure and follow head position with respect to the MEG sensors. Positions of the HPI coils, three anatomical landmarks (nasion, left and right preauricular points), and $\sim 150$ additional points on the head surface will be determined with a Fastrak 3D digitizer (Polhemus, Vermont, USA). Vertical and horizontal electro-oculograms will be recorded with two pairs of electrodes. The first pair is placed above and below the right eye and the sec2 ond pair lateral to the outer canthi of the eyes. Electrocardiography will be recorded with a pair of electrodes placed 3-5 $\mathrm{cm}$ bellow right and left clavicle and with 8 $12 \mathrm{~cm}$ inter-electrode distance. The ground electrode 2 will be placed on the right clavicle. EMG activity will be recorded from Sol, MG and TA muscles (EMG setup 3).

Equipment Two custom-made MEG-compatible devices (University of Jyväskylä, Jyväskylä, Finland) were utilized in the study: a pneumatic ankle-movement actuator and a dynamometer capable of measuring isometric ankle plantarflexion and dorsiflexion forces. The chair was modified by removing its shank and foot support and installing a metallic rail (aluminum and brass) to allow adjustable attachment of both equipment to it.

The pneumatic ankle-movement actuator was adapted from the device made by Piitulainen et al. [116]. The operating principle of the MEG compatible pneumatic actuators is reported in Piitulainen et al. [117]. In brief, the actuator is composed of four pneumatic muscles (DMSP-10-120 N-AM-CM, Festo, Vantaa, Finland) embedded into a plastic and aluminum frame with a rotating platform that supports the participant's foot. The rotation occurs in the sagittal plane (i.e. ankle dorsiflexion) when the internal pressure of the pneumatic muscles is increased (from 1 to 7 bar) and returns to the initial position when the air pressure is released. Rotation axes of the actuator and ankle joint can be aligned. The foot is fixed to the rotating platform by a strap passing firmly around the forefoot.

The isometric dynamometer composed of a metallic plate in L format, with a hard foam support for the posterior distal shank and for the heel. An adjustable metal attachment is positioned on the person's forefoot. The shank-support is embedded with a load cell and has a strap to fasten the shank, enabling it to measure isometric knee extension and flexion forces. Another load cell is embedded in a support part holding the adjustable metal attachment. A strap firmly secures the participant's forefoot and thus the load cell can measure both isometric ankle plantarflexion and dorsiflexion forces.

MEG protocol Before the participant enters the shielded room, a 5-min empty room measurement will be recorded to estimate intrinsic noise levels of the MEG sensors for the needs of later data analysis. Three experimental MEG tasks will be performed in the following order: (1) resting state, (2) proprioceptive stimulation and (3) isometric muscle action. The resting-state task consists of two 5min recordings, in which the participant will remain relaxed fixating on a cross on a screen positioned $1 \mathrm{~m}$ away from the participant's eyes. In the proprioceptive stimulation, the pneumatic ankle-movement actuator will evoke a 
total of 100 dorsiflexions (movement range from $0^{\circ}$ to $\sim$ $15^{\circ}$ ) with a moderate velocity (mean: $15^{\circ} / \mathrm{s}$; peak: $35^{\circ} / \mathrm{s}$ ) every $4 \mathrm{~s}$ with $\pm 0.2 \mathrm{~s}$ random jitter between the consecutive stimuli. Participants will be instructed to relax and not to resist the movement, fixate on the fixation cross, and avoid excessive eye and head movements. The view of the foot and actuator is blocked, and Brownian noise will be played through earplugs in an individually adjusted volume to mask the noise generated by the movement actuator. In the third task, first a preparatory activity of ten 2-s isometric dorsiflexions with increasing intensity (i.e. $\sim 20$ to $90 \%$ of $\mathrm{MVC}$ ) at $0^{\circ}$ ankle angle in the ankle dynamometer will be performed. Then, in the same position, three to five maximum isometric dorsiflexions $(\sim 3 \mathrm{~s}$ each) will be performed, followed by four sets of submaximal isometric dorsiflexions at $10 \%$ of MVC with $120 \mathrm{~s}$ of duration each. Visual torque feedback will be provided in real-time, and participants will be instructed to maintain as stable as possible torque throughout each submaximal trial. To avoid fatigue, 1-2 min of rest periods will be enforced between trials (except in the preparatory activity). If the participant is unable to perform dorsiflexion adequately, plantarflexion will be used. Continuous HPI tracking will be performed for later correction of head movements.

MEG data preprocessing First, visual data inspection will be performed to identify bad channels and periods with intense noise. Maxfilter (ver. 3.0, Elekta Neuromag Oy, Helsinki, Finland) with spatiotemporal signal space separation method [118] will be used to reduce endogenous and exogenous sources of noise in the MEG signals. Additionally, MaxFilter will be utilized for head movement compensation during MEG recording and to align the head coordinates across the different longitudinal recording sessions (pre-tests 1 and 2, and post-test 1). The coordinate transformation will be done to the participant individual mean reference head position within each MEG task separately. Artifacts caused by eye movements and cardiac activity will be identified and removed using the electro-oculograms and electrocardiography signals in combination with independent component analysis implemented in MNE-Python software package $[119,120]$.

Data analyses All further analyses of the MEG data will be processed and analyzed with the MNE-Python software package. The data will be averaged across proprioceptive stimuli in order to examine the passivemovement-evoked fields, and the respective modulation of the rhythmic activity in primary sensorimotor cortex at alpha, beta and possibly also at gamma frequency bands. Cortico-muscular and intramuscular coherence for TA will be computed as described in the EMG section. Resting state data will be analyzed with a special focus on the sensorimotor cortical network. Torque steadiness during the $8 \mathrm{~min}$ of submaximal effort will be calculated utilizing the standard deviation and coefficient of variation.

\section{Statistical analyses}

Mean/median values, standard deviations and 95\% confidence intervals will be reported. Normality of data will be tested using the Shapiro-Wilk test. General linear mixed model analysis will be utilized to study the differences between time ( 2 pre-tests and 1 or 2 post-tests) in the $\mathrm{CP}$ group. Independent $\mathrm{t}$-tests will be used to compare groups (i.e. CP vs. TD) and paired t-tests to compare the two TD testing times (pre-test 1 and 2). Pearson correlation analysis will be performed to check association between dependent variables. The interference of variables such as PA levels or maturity offset will be controlled if they have a strong linear relationship with other dependent variables. Nonparametric tests may be utilized if necessary. Significance level will be set at $P<0.05$.

\section{Discussion}

The current training intervention protocol combines different training modalities in an attempt to enhance motor function in people with $\mathrm{CP}$. The individually tailored aspects are: 1) flexibility training for muscles diagnosed short in the Pre-tests; 2) the number of training sessions and strength exercises in each session will be adjusted depending, e.g., on the participants' motivation, stamina and schedule; 3 ) introduction of a short isometric hold ( $3 \mathrm{~s})$ in case that the joint range of motion is limited; 4) stabilization procedures to ensure proper execution of the movements, e.g., using a foam ball to prevent hip adduction during leg press.

The major limitation in this study is the effect of the growth spurt on the dependent variables. If it happens during the control period, results between pre-test 1 and 2 may be considerably different, making it harder for any intervention related changes to be statistically different. If the growth spurt happens during the intervention it may increase its effectiveness, whereas if it happens during the maintenance period it may slow down the adaptation loss. By carefully monitoring the maturity indicators it will be possible to understand and estimate how each participant was affected by the maturation process. A second limitation regards the study's external validity: due to its length and great number of testing and training sessions, only participants and families that are already inclined towards higher levels of PA will likely choose to participate. Since PA levels are being measured objectively, it will be possible to characterize and compare this study with others and check this assumption. 


\section{Abbreviations}

3D: Three-Dimensional; 6MWT: Six Minutes Walking Test; CIBR: Centre For Interdisciplinary Brain Research; CP: Cerebral Palsy; DSRT: Dynamic Stretch Reflex Threshold; EMG: Electromyography; GMFCS: Gross Motor Function Classification System; GMFM: Gross Motor Function Measure; HPI: Head Position Indicator; H-Reflex: Hoffman Reflex; MEG: Magnetoencephalography; MG: Medial Gastrocnemius; MVC: Maximum Voluntary Contraction; NMRC: Neuromuscular Research Center; PA: Physical Activity; PAD: PostActivation Depression; RCT: Randomized Controlled Trial; RMS: Root Mean Square; ROM: Range of Motion; S1: Testing Session One; S2: Testing Session Two; S3: Testing Session Three; Sol: Soleus; TA: Tibialis Anterior; TD: Typically Developing; TSRT: Tonic Stretch Reflex Threshold

\section{Acknowledgements}

We would like to thank all personnel involved in building the two equipment utilized in the magnetoencephalography measurements. The force dynamometer was designed by Simon Walker and Janne Avela, and built by Sirpa Roivas, Jouni Tukiainen and Markku Ruuskanen, all from NMRC. The pneumatic actuator was built by Viki-Veikko Elomaa (CIBR). Technical support to design the pneumatic actuators was provided by Helge Kainulainen and Veikko Jousmäki both from Aalto Neurolmaging, Aalto University.

\section{Authors' contributions}

$P V, H P, E A H, T P, J A$ and TF defined the study protocol. PV and TF developed the training protocol and PV is responsible for implementing it. PV is responsible for data collection. TF is the chief investigator for the study. All authors will be involved in preparing the manuscripts for publication according to the Vancouver protocol. All authors have read and approved the final manuscript.

\section{Funding}

This work was supported by the Olvi Foundation, University of Jyväskylä, Academy of Finland (grants \#296240, \#307250 and \#327288) and Jane and Aatos Erkko Foundation. None of the funding organizations participated in the creation of the study design, data collection, data analyses or writing the manuscript.

\section{Availability of data and materials}

Not applicable.

\section{Ethical approval and consent to participate}

Ethical approval has been granted by the ethics committee of the Central Finland Healthcare District (U8/2017) and the study has been registered prospectively in the International Standard Randomized Controlled Trial (ISRC TN69044459). Two minor protocol amendments were also approved (2018/ 2019) and the registered trial information was updated accordingly. Prospective participants will come to the Neuromuscular Research Center (NMRC) at the University of Jyväskylä, where a detailed overview of the project will be given. All individuals who are willing to participate, and who meet the inclusion criteria (assessed by a screening questionnaire), will sign the informed consent to participate in the study. Legal guardians will be asked to sign the informed consent for children under 18 years, and written assent from children will also be required. Participation to this study is voluntary, all participants are informed that they can withdraw from the study at any time without consequences. The study will comply with the Declaration of Helsinki. Any adverse effects caused by the intervention will be reported to the ethics committee within 15 days, and later reported in the publications.

\section{Consent for publication}

Not applicable.

\section{Competing interests}

The authors declare that they have no competing interests.

\section{Author details}

'Neuromuscular Research Center, Faculty of Sport and Health Sciences, University of Jyväskylä, Jyväskylä, Finland. ${ }^{2}$ Department of Neuroscience and Biomedical Engineering, Aalto University, Espoo, Finland. ${ }^{3}$ Institute of Biomedicine, School of Medicine, University of Eastern Finland, Kuopio,
Finland. ${ }^{4}$ Centre for Interdisciplinary Brain Research, University of Jyväskylä, Jyväskylä, Finland.

Received: 23 July 2020 Accepted: 5 February 2021

Published online: 26 February 2021

\section{References}

1. Rosenbaum P, Paneth N, Leviton A, Goldstein M, Bax M, Damiano D, et al. A report: the definition and classification of cerebral palsy April 2006. Dev Med Child Neurol Suppl. 2007 Feb;109:8-14.

2. Kirby RS, Wingate MS, Van Naarden BK, Doernberg NS, Arneson CL, Benedict $R E$, et al. Prevalence and functioning of children with cerebral palsy in four areas of the United States in 2006: a report from the autism and developmental disabilities monitoring network. Res Dev Disabil. 2011;32(2): 462-9.

3. Surveillance of Cerebral Palsy in Europe. Surveillance of cerebral palsy in Europe: a collaboration of cerebral palsy surveys and registers. Surveillance of Cerebral Palsy in Europe (SCPE). Dev Med Child Neurol. 2000;42(12):816-24.

4. Winter S, Autry A, Boyle C, Yeargin-Allsopp M. Trends in the prevalence of cerebral palsy in a population-based study. Pediatrics. 2002;110(6):1220-5.

5. van DN B-OL, Aertbeliën E, Bonikowski M, Braendvik SM, Broström EW, et al. European consensus on the concepts and measurement of the pathophysiological neuromuscular responses to passive muscle stretch. Eur J Neurol. 2017:24(7):981-e38.

6. Geertsen SS, Kirk H, Lorentzen J, Jorsal M, Johansson CB, Nielsen JB. Impaired gait function in adults with cerebral palsy is associated with reduced rapid force generation and increased passive stiffness. Clin Neurophysiol. 2015 Dec;126(12):2320-9.

7. Lieber RL, Steinman S, Barash IA, Chambers H. Structural and functional changes in spastic skeletal muscle. Muscle Nerve. 2004 May;29(5):615-27.

8. Sinkjaer T, Magnussen I. Passive, intrinsic and reflex-mediated stiffness in the ankle extensors of hemiparetic patients. Brain. 1994;117(Pt 2):355-63.

9. Farmer SE, James M. Contractures in orthopaedic and neurological conditions: a review of causes and treatment. Disabil Rehabil. 2001;23(13): $549-58$.

10. Gage JR, Novacheck TF. An update on the treatment of gait problems in cerebral palsy. J Pediatr Orthop B. 2001;10(4):265-74.

11. Lieber RL, Friden J. Muscle contracture and passive mechanics in cerebral palsy. J Appl Physiol(1985). 2019;126(5):1492-501.

12. Tedroff $K$, Granath F, Forssberg H, Haglund-Akerlind Y. Long-term effects of botulinum toxin a in children with cerebral palsy. Dev Med Child Neurol. 2009 Feb;51(2):120-7.

13. Tedroff $\mathrm{K}$, Lowing $\mathrm{K}$, Jacobson DN, Astrom E. Does loss of spasticity matter? A 10-year follow-up after selective dorsal rhizotomy in cerebral palsy. Dev Med Child Neurol. 2011:53(8):724-9.

14. Gough M, Shortland AP. Could muscle deformity in children with spastic cerebral palsy be related to an impairment of muscle growth and altered adaptation? Dev Med Child Neurol. 2012;54(6):495-9.

15. Willerslev-Olsen $M$, Choe Lund $M$, Lorentzen J, Barber L, Kofoed-Hansen M, Nielsen JB. Impaired muscle growth precedes development of increased stiffness of the triceps surae musculotendinous unit in children with cerebral palsy. Dev Med Child Neurol. 2018;60(7):672-9.

16. Garcia CC, Alcocer-Gamboa A, Ruiz MP, Caballero IM, Faigenbaum AD, Esteve-Lanao J, et al. Metabolic, cardiorespiratory, and neuromuscular fitness performance in children with cerebral palsy: a comparison with healthy youth. J Exerc Rehabil. 2016;12(2):124-31.

17. Moreau NG, Falvo MJ, Damiano DL. Rapid force generation is impaired in cerebral palsy and is related to decreased muscle size and functional mobility. Gait Posture. 2012;35(1):154-8.

18. Wiley ME, Damiano DL. Lower-extremity strength profiles in spastic cerebral palsy. Dev Med Child Neurol. 1998;40(2):100-7.

19. Russell DJ, Avery LM, Rosenbaum PL, Raina PS, Walter SD, Palisano RJ. Improved scaling of the gross motor function measure for children with cerebral palsy: evidence of reliability and validity. Phys Ther. 2000;80(9):873-85.

20. Hanna SE, Rosenbaum PL, Bartlett DJ, Palisano RJ, Walter SD, Avery L, et al. Stability and decline in gross motor function among children and youth with cerebral palsy aged 2 to 21 years. Dev Med Child Neurol. 2009;51(4): 295-302.

21. Smits DW, Gorter JW, Hanna SE, Dallmeijer AJ, van Eck M, Roebroeck ME, et al. Longitudinal development of gross motor function among Dutch 
children and young adults with cerebral palsy: an investigation of motor growth curves. Dev Med Child Neurol. 2013;55(4):378-84.

22. Carlon SL, Taylor NF, Dodd KJ, Shields N. Differences in habitual physical activity levels of young people with cerebral palsy and their typically developing peers: a systematic review. Disabil Rehabil. 2013;35(8):647-55.

23. Nieuwenhuijsen $\mathrm{C}$, van der Slot WM, Beelen A, Arendzen JH, Roebroeck ME, Stam HJ, et al. Inactive lifestyle in adults with bilateral spastic cerebral palsy. J Rehabil Med. 2009;41(5):375-81.

24. Maltais DB, Wiart L, Fowler E, Verschuren O, Damiano DL. Health-related physical fitness for children with cerebral palsy. J Child Neurol. 2014;29(8): 1091-100.

25. Verschuren $\mathrm{O}$, Peterson MD, Balemans AC, Hurvitz EA. Exercise and physical activity recommendations for people with cerebral palsy. Dev Med Child Neurol. 2016;58(8):798-808

26. Bottos M, Feliciangeli A, Sciuto L, Gericke C, Vianello A. Functional status of adults with cerebral palsy and implications for treatment of children. Dev Med Child Neurol. 2001;43(8):516-28.

27. Jahnsen R, Villien L, Egeland T, Stanghelle JK, Holm I. Locomotion skills in adults with cerebral palsy. Clin Rehabil. 2004;18(3):309-16.

28. Gillett JG, Lichtwark GA, Boyd RN, Barber LA. Functional Capacity in Adults With Cerebral Palsy: Lower Limb Muscle Strength Matters. Arch Phys Med Rehabil. 2018;99(5):900-906.e1.

29. Ross SA, Engsberg JR. Relation between spasticity and strength in individuals with spastic diplegic cerebral palsy. Dev Med Child Neurol. 2002; 44(3):148-57.

30. Garber CE, Blissmer B, Deschenes MR, Franklin BA, Lamonte MJ, Lee IM, et al. American College of Sports Medicine position stand. Quantity and quality of exercise for developing and maintaining cardiorespiratory, musculoskeletal, and neuromotor fitness in apparently healthy adults: guidance for prescribing exercise. Med Sci Sports Exerc. 2011;43(7):1334-59.

31. Faigenbaum AD, Kraemer WJ, Blimkie CJ, Jeffreys I, Micheli L, Nitka M, et al. Youth resistance training: updated position statement paper from the national strength and conditioning association. J Strength Cond Res. 2009; 23(5 Suppl):S60-79.

32. Gillett JG, Lichtwark GA, Boyd RN, Barber LA. Functional anaerobic and strength training in young adults with cerebral palsy. Med Sci Sports Exerc. 2018b;50(8):1549-57.

33. Kirk H, Geertsen SS, Lorentzen J, Krarup KB, Bandholm T, Nielsen JB. Explosive resistance training increases rate of force development in ankle Dorsiflexors and gait function in adults with cerebral palsy. J Strength Cond Res. 2016;30(10):2749-60

34. MacPhail HE, Kramer JF. Effect of isokinetic strength-training on functional ability and walking efficiency in adolescents with cerebral palsy. Dev Med Child Neurol. 1995;37(9):763-75.

35. Moreau NG, Holthaus K, Marlow N. Differential adaptations of muscle architecture to high-velocity versus traditional strength training in cerebral palsy. Neurorehabil Neural Repair. 2013;27(4):325-34.

36. Taylor NF, Dodd KJ, Baker RJ, Willoughby K, Thomason P, Graham HK. Progressive resistance training and mobility-related function in young people with cerebral palsy: a randomized controlled trial. Dev Med Child Neurol. 2013;55(9):806-12.

37. Scholtes VA, Becher JG, Comuth A, Dekkers H, Van Dijk L, Dallmeijer AJ. Effectiveness of functional progressive resistance exercise strength training on muscle strength and mobility in children with cerebral palsy: a randomized controlled trial. Dev Med Child Neurol. 2010;52(6):e107-13.

38. Scholtes VA, Becher JG, Janssen-Potten YJ, Dekkers H, Smallenbroek L, Dallmeijer AJ. Effectiveness of functional progressive resistance exercise training on walking ability in children with cerebral palsy: a randomized controlled trial. Res Dev Disabil. 2012;33(1):181-8.

39. Novak I, Morgan C, Fahey M, Finch-Edmondson M, Galea C, Hines A, et al. State of the evidence traffic lights 2019: systematic review of interventions for preventing and treating children with cerebral palsy. Current Neurology and Neuroscience Reports. 2020;20(2):3.

40. Park EY, Kim WH. Meta-analysis of the effect of strengthening interventions in individuals with cerebral palsy. Res Dev Disabil. 2014;35(2):239-49.

41. Folland JP, Williams AG. The adaptations to strength training: morphological and neurological contributions to increased strength. Sports Med. 2007:37(2):145-68.

42. Damiano $\mathrm{DL}$, Vaughan $\mathrm{CL}$, Abel MF. Muscle response to heavy resistance exercise in children with spastic cerebral palsy. Dev Med Child Neurol. 1995; 37(8):731-9.
43. Malhotra S, Pandyan AD, Day CR, Jones PW, Hermens H. Spasticity, an impairment that is poorly defined and poorly measured. Clin Rehabil. 2009; 23(7):651-8

44. Bohannon RW, Smith MB. Interrater reliability of a modified Ashworth scale of muscle spasticity. Phys Ther. 1987;67(2):206-7.

45. Pandyan AD, Johnson GR, Price Cl, Curless RH, Barnes MP, Rodgers H. A review of the properties and limitations of the Ashworth and modified Ashworth scales as measures of spasticity. Clin Rehabil. 1999;13(5):373-83.

46. Mockford M, Caulton JM. Systematic review of progressive strength training in children and adolescents with cerebral palsy who are ambulatory. Pediatr Phys Ther. 2008:20(4):318-33.

47. Harvey LA, Katalinic OM, Herbert RD, Moseley AM, Lannin NA, Schurr K. Stretch for the treatment and prevention of contracture: an abridged republication of a Cochrane systematic review. J Physiother. 2017;63(2):6775.

48. Pin T, Dyke P, Chan M. The effectiveness of passive stretching in children with cerebral palsy. Dev Med Child Neurol. 2006;48(10):855-62.

49. Wiart L, Darrah J, Kembhavi G. Stretching with children with cerebral palsy: what do we know and where are we going? Pediatr Phys Ther. 2008;20(2): 173-8.

50. Theis $\mathrm{N}$, Korff $\mathrm{T}$, Kairon $\mathrm{H}$, Mohagheghi AA. Does acute passive stretching increase muscle length in children with cerebral palsy? Clin Biomech (Bristol, Avon). 2013;28(9-10):1061-7.

51. Theis N, Korff T, Mohagheghi AA. Does long-term passive stretching alter muscle-tendon unit mechanics in children with spastic cerebral palsy? Clin Biomech (Bristol, Avon). 2015;30(10):1071-6.

52. Zhao H, Wu YN, Hwang M, Ren Y, Gao F, Gaebler-Spira D, et al. Changes of calf muscle-tendon biomechanical properties induced by passive-stretching and active-movement training in children with cerebral palsy. J Appl Physiol (1985). 2011;111(2):435-42

53. Rodda J, Graham HK. Classification of gait patterns in spastic hemiplegia and spastic diplegia: a basis for a management algorithm. Eur J Neurol. 2001;8(Suppl 5):98-108.

54. Booth ATC, Buizer Al, Meyns P, Oude Lansink ILB, Steenbrink F, van der Krogt MM. The efficacy of functional gait training in children and young adults with cerebral palsy: a systematic review and meta-analysis. Dev Med Child Neurol. 2018;60(9):866-83.

55. Tae HJ, Hyun KY, Won PJ, Mo KH, Seok NK. Three-Dimensional Kinematic Analysis during Upslope Walking with Different Inclinations by Healthy Adults. J Physical Therap Sci. 2009;21(4):388.

56. Leroux A, Fung J, Barbeau H. Adaptation of the walking pattern to uphill walking in normal and spinal-cord injured subjects. Exp Brain Res. 1999; 126(3):359-68.

57. Lorentzen J, Kirk H, Fernandez-Lago H, Frisk R, Scharff Nielsen N, Jorsal M, et al. Treadmill training with an incline reduces ankle joint stiffness and improves active range of movement during gait in adults with cerebral palsy. Disabil Rehabil. 2017;39(10):987-93.

58. Willerslev-Olsen M, Lorentzen J, Nielsen JB. Gait training reduces ankle joint stiffness and facilitates heel strike in children with cerebral palsy. NeuroRehabilitation. 2014;35(4):643-55.

59. Graham JE, Karmarkar AM, Ottenbacher KJ. Small sample research designs for evidence-based rehabilitation: issues and methods. Arch Phys Med Rehabil. 2012:93(8 Suppl):S111-6.

60. Hawkins NG, Sanson-Fisher RW, Shakeshaft A, D'Este C, Green LW. The multiple baseline design for evaluating population-based research. Am J Prev Med. 2007;33(2):162-8.

61. Biglan A, Ary D, Wagenaar AC. The value of interrupted time-series experiments for community intervention research. Prev Sci. 2000;1 (1):31-49.

62. Palisano R, Rosenbaum P, Walter S, Russell D, Wood E, Galuppi B. Development and reliability of a system to classify gross motor function in children with cerebral palsy. Dev Med Child Neurol. 1997; 39(4):214-23.

63. Maher CA, Williams MT, Olds TS. The six-minute walk test for children with cerebral palsy. Int J Rehabil Res. 2008;31(2):185-8.

64. Verschuren O, Ketelaar M, Keefer D, Wright V, Butler J, Ada L, et al. Identification of a core set of exercise tests for children and adolescents with cerebral palsy: a Delphi survey of researchers and clinicians. Developmental Med Child Neurol. 2011;53(5):449-56.

65. Verschuren O, Balemans AC. Update of the core set of exercise tests for children and adolescents with cerebral palsy. Pediatr Phys Ther. 2015;27(2): 187-9. 
66. Hopkins WG. Estimating sample size for magnitude-based inferences. Sportscience. 2006;10:63-70.

67. Tanimoto M, Ishii N. Effects of low-intensity resistance exercise with slow movement and tonic force generation on muscular function in young men. J Appl Physiol (1985). 2006;100(4):1150-7.

68. Lieber RL, Boakes JL. Sarcomere length and joint kinematics during torque production in frog hindlimb. Am J Phys. 1988;254(6 Pt 1):C759-68.

69. de Bruin M, Smeulders MJ, Kreulen M. Why is joint range of motion limited in patients with cerebral palsy? J Hand Surg Eur Vol. 2013 Jan;38(1):8-13.

70. Graves JE, Pollock ML, Jones AE, Colvin AB, Leggett SH. Specificity of limited range of motion variable resistance training. Med Sci Sports Exerc. 1989; 21(1):84-9.

71. Rhea MR, Kenn JG, Peterson MD, Massey D, Simão R, Marin PJ, et al. JointAngle Specific Strength Adaptations Influence Improvements in Power in Highly Trained Athletes. Human Movement. 2016;17(1):43-9.

72. Harvey D. Assessment of the flexibility of elite athletes using the modified Thomas test. Br J Sports Med. 1998;32(1):68-70.

73. Costa M, Goldberger AL, Peng CK. Multiscale entropy analysis of complex physiologic time series. Phys Rev Lett. 2002;89(6):068102.

74. Wu S, Wu C, Lin S, Lee K, Peng C. Analysis of complex time series using refined composite multiscale entropy. Physics Lett A. 2014;378(20):1369-74.

75. Aziz W, Arif M. Multiscale permutation entropy of physiological time series. Pakistan Section Multitopic Conference, Karachi, Pakistan. 2005. p. 1-6. https://doi.org/10.1109/INMIC.2005.334494.

76. Li D, Li X, Liang Z, Voss LJ, Sleigh JW. Multiscale permutation entropy analysis of EEG recordings during sevoflurane anesthesia. J Neural Eng. 2010;7(4):046010-2560 Epub 2010 Jun 28.

77. Ihlen EAF, Weiss A, Bourke A, Helbostad JL, Hausdorff JM. The complexity of daily life walking in older adult community-dwelling fallers and non-fallers. J Biomech. 2016;49(9):1420-8.

78. Richman JS, Randall Moorman J. Physiological time-series analysis using approximate entropy and sample entropy. Am J Physiol Heart Circ Physiol. 2000;278(6):H2039-49.

79. Zhang T, Yang Z, Coote JH. Cross-sample entropy statistic as a measure of complexity and regularity of renal sympathetic nerve activity in the rat. Exp Physiol. 2007;92(4):659-69.

80. Davis RB, Õunpuu S, Tyburski D, Gage JR. A gait analysis data collection and reduction technique. Human Movement Sci. 1991:10(5):575-87.

81. Kadaba MP, Ramakrishnan HK, Wootten ME. Measurement of lower extremity kinematics during level walking. J Orthop Res. 1990:8(3): 383-92.

82. Delp SL, Anderson FC, Arnold AS, Loan P, Habib A, John CT, et al. OpenSim: open-source software to create and analyze dynamic simulations of movement. IEEE Trans Biomed Eng. 2007:54(11):1940-50.

83. Brockett CL, Morgan DL, Proske U. Human hamstring muscles adapt to eccentric exercise by changing optimum length. Med Sci Sports Exerc 2001;33(5):783-90.

84. Reid S, Hamer P, Alderson J, Lloyd D. Neuromuscular adaptations to eccentric strength training in children and adolescents with cerebral palsy. Dev Med Child Neurol. 2010;52(4):358-63.

85. Hermens HJ, Freriks B, Disselhorst-Klug C, Rau G. Development of recommendations for SEMG sensors and sensor placement procedures. J Electromyogr Kinesiol. 2000;10(5):361-74.

86. Lamy JC, Wargon I, Mazevet D, Ghanim Z, Pradat-Diehl P, Katz R. Impaired efficacy of spinal presynaptic mechanisms in spastic stroke patients. Brain. 2009;132(Pt 3):734-48.

87. Yang Y, Xiao J, Song W. Post-activation depression of the lower extremities in stroke patients with spasticity and spastic equinovarus deformity. Arq Neuropsiquiatr. 2015;73(6):493-8.

88. Blanchette AK, Mullick AA, Moin-Darbari K, Levin MF. Tonic stretch reflex threshold as a measure of ankle plantar-flexor spasticity after stroke. Phys Ther. 2016:96(5):687-95.

89. Calota A, Feldman AG, Levin MF. Spasticity measurement based on tonic stretch reflex threshold in stroke using a portable device. Clin Neurophysiol. 2008;119(10):2329-37.

90. Boonstra TW, Breakspear M. Neural mechanisms of intermuscular coherence: implications for the rectification of surface electromyography. J Neurophysiol. 2012;107(3):796-807.

91. Ward NJ, Farmer SF, Berthouze L, Halliday DM. Rectification of EMG in low force contractions improves detection of motor unit coherence in the betafrequency band. J Neurophysiol. 2013;110(8):1744-50.
92. Farina D, Merletti R, Enoka RM. The extraction of neural strategies from the surface EMG: an update. J Appl Physiol (1985). 2014;117(11):1215-30.

93. Halliday DM, Rosenberg JR, Amjad AM, Breeze P, Conway BA, Farmer SF. A framework for the analysis of mixed time series/point process data-theory and application to the study of physiological tremor, single motor unit discharges and electromyograms. Prog Biophys Mol Biol. 1995;64(2-3):237-78.

94. Gardner WA. A unifying view of coherence in signal processing. Signal Processing 1992. 1992;29(2):113-40.

95. Amjad AM, Halliday DM, Rosenberg JR, Conway BA. An extended difference of coherence test for comparing and combining several independent coherence estimates: theory and application to the study of motor units and physiological tremor. J Neurosci Methods. 1997;73(1):69-79.

96. Halliday DM, Rosenberg JR. On the application, estimation and interpretation of coherence and pooled coherence. J Neurosci Methods. 2000:100(1-2):173-4

97. Kendall FP, McCreary EK, Provance PG, Rodgers MM, Romani WA. Muscles: Testing and function with posture and pain. 5th ed. Baltimore: Lippincott Williams \& Wilkins; 2005

98. Clapis PA, Davis SM, Davis RO. Reliability of inclinometer and goniometric measurements of hip extension flexibility using the modified Thomas test. Physiother Theory Pract. 2008;24(2):135-41.

99. Gabbe BJ, Bennell KL, Wajswelner H, Finch CF. Reliability of common lower extremity musculoskeletal screening tests. Physical Therapy in Sport 2004. 2004;5(2):90-7.

100. Bandy WD, Irion JM, Briggler M. The effect of time and frequency of static stretching on flexibility of the hamstring muscles. Phys Ther. 1997;77(10): 1090-6.

101. Gnat R, Kuszewski M, Koczar R, Dziewonska A. Reliability of the passive knee flexion and extension tests in healthy subjects. J Manip Physiol Ther. 2010; 33(9):659-65.

102. Baumbach SF, Brumann M, Binder J, Mutschler W, Regauer M, Polzer H. The influence of knee position on ankle dorsiflexion - a biometric study. BMC Musculoskeletal Disord. 2014;15:246.

103. Moseley AM, Crosbie J, Adams R. Normative data for passive ankle plantarflexion--dorsiflexion flexibility. Clin Biomech (Bristol, Avon). 2001;16(6): $514-21$.

104. Jackson AS, Blair SN, Mahar MT, Wier LT, Ross RM, Stuteville JE. Prediction of functional aerobic capacity without exercise testing. Med Sci Sports Exerc. 1990;22(6):863-70

105. Mitchell LE, Ziviani J, Boyd RN. Variability in measuring physical activity in children with cerebral palsy. Med Sci Sports Exerc. 2015;47(1):194-200.

106. Brond JC, Andersen LB, Arvidsson D. Generating ActiGraph counts from raw acceleration recorded by an alternative monitor. Med Sci Sports Exerc. 2017; 49(11):2351-60.

107. Trost SG, Fragala-Pinkham M, Lennon N, O'Neil ME. Decision trees for detection of activity intensity in youth with cerebral palsy. Med Sci Sports Exerc. 2016;48(5):958-66.

108. Finni T, Hu M, Kettunen P, Vilavuo T, Cheng S. Measurement of EMG activity with textile electrodes embedded into clothing. Physiol Meas. 2007;28(11): 1405-19.

109. Pesola AJ, Laukkanen A, Tikkanen O, Sipila S, Kainulainen H, Finni T. Muscle inactivity is adversely associated with biomarkers in physically active adults. Med Sci Sports Exerc. 2015;47(6):1188-96.

110. Gao Y, Melin M, Makarainen K, Rantalainen T, Pesola AJ, Laukkanen A, et al. Children's physical activity and sedentary time compared using assessments of accelerometry counts and muscle activity level. PeerJ. 2018;6:e5437.

111. Russell DJ, Rosenbaum PL, Wright M, Avery LM. Gross motor function measure (GMFM-66 and GMFM-88) User's manual. 2nd ed. London: Mac Keith Press; 2013.

112. Moore SA, McKay HA, Macdonald H, Nettlefold L, Baxter-Jones AD, Cameron $\mathrm{N}$, et al. Enhancing a somatic maturity prediction model. Med Sci Sports Exerc. 2015;47(8):1755-64.

113. Tompuri TT, Lakka TA, Hakulinen M, Lindi V, Laaksonen DE, Kilpelainen TO, et al. Assessment of body composition by dual-energy X-ray absorptiometry, bioimpedance analysis and anthropometrics in children: the physical activity and nutrition in children study. Clin Physiol Funct Imaging. 2015;35(1):21-33.

114. Matthews DR, Hosker JP, Rudenski AS, Naylor BA, Treacher DF, Turner RC. Homeostasis model assessment: insulin resistance and beta-cell function from fasting plasma glucose and insulin concentrations in man Diabetologia. 1985;28(7):412-9. 
115. Haapala EA, Veijalainen A, Kujala UM, Finni T. Reproducibility of pulse wave velocity and augmentation index derived from non-invasive occlusive oscillometric tonometry analysis in adolescents. Clin Physiol Funct Imaging. 2019:39(1):22-8

116. Piitulainen H, Seipäjärvi S, Avela J, Parviainen T, Walker S. Cortical Proprioceptive Processing Is Altered by Aging. Front Aging Neurosci. 2018. 10:147.

117. Piitulainen H, Bourguignon M, Hari R, Jousmaki V. MEG-compatible pneumatic stimulator to elicit passive finger and toe movements. Neuroimage. 2015;112:310-7.

118. Taulu S, Simola J. Spatiotemporal signal space separation method for rejecting nearby interference in MEG measurements. Phys Med Biol. 2006; 51(7):1759-68.

119. Gramfort A, Luessi M, Larson E, Engemann DA, Strohmeier D, Brodbeck C, et al. MEG and EEG data analysis with MNE-python. Front Neurosci. 2013;7: 267.

120. Gramfort A, Luessi M, Larson E, Engemann DA, Strohmeier D, Brodbeck C, et al. MNE software for processing MEG and EEG data. Neuroimage. 2014;86: $446-60$.

\section{Publisher's Note}

Springer Nature remains neutral with regard to jurisdictional claims in published maps and institutional affiliations.

Ready to submit your research? Choose BMC and benefit from:

- fast, convenient online submission

- thorough peer review by experienced researchers in your field

- rapid publication on acceptance

- support for research data, including large and complex data types

- gold Open Access which fosters wider collaboration and increased citations

- maximum visibility for your research: over $100 \mathrm{M}$ website views per year

At $\mathrm{BMC}$, research is always in progress.

Learn more biomedcentral.com/submissions 